\title{
Short parietal lobe connections of the human and monkey brain
}

Catani, Marco; Robertsson, Naianna; Beyh, Ahmad; Huynh, Vincent; de Santiago Requejo, Francisco; Howells, Henrietta; Barrett, Rachel L. C.; Aiello, Marco; Cavaliere, Carlo; Dyrby, Tim Bjørn

Total number of authors:

15

Published in:

Cortex

Link to article, DOI:

10.1016/j.cortex.2017.10.022

Publication date:

2017

Document Version

Peer reviewed version

Link back to DTU Orbit

Citation (APA):

Catani, M., Robertsson, N., Beyh, A., Huynh, V., de Santiago Requejo, F., Howells, H., Barrett, R. L. C., Aiello, M., Cavaliere, C., Dyrby, T. B., Krug, K., Ptito, M., D'Arceuil, H., Forkel, S. J., \& Dell'Acqua, F. (2017). Şhort parietal lobe connections of the human and monkey brain. Cortex, 97, 339-357.

https://doi.org/10.1016/j.cortex.2017.10.022

\section{General rights}

Copyright and moral rights for the publications made accessible in the public portal are retained by the authors and/or other copyright owners and it is a condition of accessing publications that users recognise and abide by the legal requirements associated with these rights.

- Users may download and print one copy of any publication from the public portal for the purpose of private study or research.

- You may not further distribute the material or use it for any profit-making activity or commercial gain

- You may freely distribute the URL identifying the publication in the public portal 


\title{
Special issue: Research report
}

\section{Short parietal lobe connections of the human and monkey brain}

\author{
Marco Catani ${ }^{a, b, *}$, Naianna Robertsson ${ }^{a, b}$, Ahmad Beyh ${ }^{a, b}$, \\ Vincent Huynh ${ }^{a, b, c}$, Francisco de Santiago Requejo ${ }^{a, b}$, \\ Henrietta Howells ${ }^{a, b}$, Rachel L.C. Barrett ${ }^{a, b}$, Marco Aiello ${ }^{d}$, \\ Carlo Cavaliere $^{d}$, Tim B. Dyrby ${ }^{e, f}$, Kristine Krug ${ }^{g}$, Maurice Ptito ${ }^{h, i}$, \\ Helen D'Arceuil ${ }^{j}$, Stephanie J. Forkel ${ }^{a, b, 1}$ and Flavio Dell'Acqua ${ }^{a, b, 1}$ \\ ${ }^{a}$ NatBrainLab, Department of Neuroimaging, Institute of Psychiatry, Psychology and Neuroscience, King's College \\ London, London, UK \\ ${ }^{\mathrm{b}}$ NatBrainLab, Sackler Institute for Translational Neurodevelopment, Department of Forensic and \\ Neurodevelopmental Science, Institute of Psychiatry, Psychology and Neuroscience, King's College London, London, \\ UK \\ c Spinal Cord Injury Center, Research, University of Zurich, Balgrist University Hospital, Zurich, Switzerland \\ d NAPLab, IRCCS SDN Istituto di Ricerca Diagnostica e Nucleare, Naples, Italy \\ e Danish Research Centre for Magnetic Resonance, Centre for Functional and Diagnostic Imaging and Research, \\ Copenhagen University Hospital Huidoure, Huidoure, Denmark \\ ${ }^{\mathrm{f}}$ Department of Applied Mathematics and Computer Science, Technical University of Denmark, Kongens Lyngby, \\ Denmark \\ g Department of Physiology, Anatomy and Genetics, University of Oxford, Oxford, UK \\ ${ }^{\mathrm{h}}$ Laboratory of Neuropsychiatry, Psychiatric Centre Copenhagen, Copenhagen, Denmark \\ ${ }^{\mathrm{i}}$ Ecole d'Optométrie, Université de Montréal, Montréal, Québec, Canada \\ ${ }^{j}$ Athinoula A. Martinos Center, Massachusetts General Hospital, Charlestown, USA
}

\section{A R T I C L E I N F O}

Article history:

Received 27 September 2017

Reviewed 2 October 2017

Revised 26 October 2017

Accepted 28 October 2017

Published online xxx

\section{Keywords:}

Parietal lobe

White matter

Diffusion tractography

\begin{abstract}
A B S T R A C T
The parietal lobe has a unique place in the human brain. Anatomically, it is at the crossroad between the frontal, occipital, and temporal lobes, thus providing a middle ground for multimodal sensory integration. Functionally, it supports higher cognitive functions that are characteristic of the human species, such as mathematical cognition, semantic and pragmatic aspects of language, and abstract thinking. Despite its importance, a comprehensive comparison of human and simian intraparietal networks is missing.

In this study, we used diffusion imaging tractography to reconstruct the major intralobar parietal tracts in twenty-one datasets acquired in vivo from healthy human subjects and eleven ex vivo datasets from five vervet and six macaque monkeys. Three regions of interest (postcentral gyrus, superior parietal lobule and inferior parietal lobule) were used to identify the tracts. Surface projections were reconstructed for both species and results
\end{abstract}

\footnotetext{
* Corresponding author. NatBrainLab, PO50 Department of Forensic and Neurodevelopmental Science, Institute of Psychiatry, Psychology and Neuroscience, King's College London, London, SE5 8AF, UK.

E-mail address: m.catani@iop.kcl.ac.uk (M. Catani).

1 These authors have contributed equally to the study. https://doi.org/10.1016/j.cortex.2017.10.022 0010-9452/@ 2017 Published by Elsevier Ltd.
} 
Pathways

Networks compared to identify similarities or differences in tract anatomy (i.e., trajectories and cortical projections). In addition, post-mortem dissections were performed in a human brain.

The largest tract identified in both human and monkey brains is a vertical pathway between the superior and inferior parietal lobules. This tract can be divided into an anterior (supramarginal gyrus) and a posterior (angular gyrus) component in both humans and monkey brains. The second prominent intraparietal tract connects the postcentral gyrus to both supramarginal and angular gyri of the inferior parietal lobule in humans but only to the supramarginal gyrus in the monkey brain. The third tract connects the postcentral gyrus to the anterior region of the superior parietal lobule and is more prominent in monkeys compared to humans. Finally, short U-shaped fibres in the medial and lateral aspects of the parietal lobe were identified in both species. A tract connecting the medial parietal cortex to the lateral inferior parietal cortex was observed in the monkey brain only.

Our findings suggest a consistent pattern of intralobar parietal connections between humans and monkeys with some differences for those areas that have cytoarchitectonically distinct features in humans. The overall pattern of intraparietal connectivity supports the special role of the inferior parietal lobule in cognitive functions characteristic of humans.

(C) 2017 Published by Elsevier Ltd.

\section{Introduction}

Despite proposed functional homologies (Culham \& Kanwisher, 2001), studies comparing the anatomy of the human and monkey brain have suggested differences between these two species in some regions of the parietal lobe (Critchley, 1953; Geschwind, 1965; Scheperjans et al., 2008). Earlier studies had already come to this conclusion by comparing the surface anatomy of the parietal sulci and measuring the extension of the gyral cortex comprised in between the folding (Papez, 1929). A major problem of this approach is the lack of correspondence between sulcal anatomy and the functional delimitation of specialised areas. Further, some of the human sulci, such as the postcentral sulcus, are absent or barely visible in brains of different monkey species (Bonin \& Bailey, 1947).

Divisions of the parietal cortex into fields or areas based on cytoarchitectonics have provided a viable and necessary alternative. Initial studies showed interspecies differences that were particularly significant for the inferior parietal lobule (Campbell, 1905). Recent findings using an operatorindependent parcellation approach have found more interspecies analogies in the inferior parietal cortex (Caspers et al., 2006) but also differences in the superior parietal lobule (Scheperjans et al., 2008). These discrepancies in the results may depend on different methods utilised to define boundaries between areas. There is however, an even more concerning limitation of this approach when differences in cytoarchitectonic variations are used to imply functional specialisation. While this is certainly true for grosser types of cellular difference (e.g., granular us agranular), the same cannot be assumed for all cytoarchitectonic distinctions (Critchley, 1953). For example, in the human brain, area 40 has different functions in the left and right hemisphere. Clearly, anatomical homologies do not necessarily imply functional homologies within a species, let alone across species. This limitation has led many anatomists to pursue another approach to comparative anatomy based on the delineation of the connectivity pattern of cortical areas (Jones \& Powell, 1970).

The first studies in monkey brains used methods for staining degenerating fibres following a cortical lesion (e.g., Marchi's or Nauta's method) and physiological neuronography (e.g., strychninization and recording of amplified impulses) to demonstrate the existence of short connections between distinct parietal regions (Bonin \& Bailey, 1947; Jones \& Powell, 1969; Warren, 1944). These results were directly transposed to humans based on the assumption of great similarity between the brains of the two species (Bailey \& Bonin, 1951). The development of axonal tracing methods led to a more detailed description of intralobar parietal connectivity in the monkey brain (Cavada \& Goldman-Rakic, 1989; Pandya \& Seltzer, 1982). The pioneering work of Pandya and Seltzer (1982) has identified two parallel main streams of intrinsic connections in the parietal lobe of the rhesus monkey: a dorsal stream of short connections between the postcentral gyrus (area 2) and the dorsal (PE, PEc) and medial (PGm) parietal cortex, and a ventral stream between postcentral gyrus and inferior parietal cortex (PF, PFG, PG, Opt). The two streams seem to be weakly interconnected, except for the most posterior regions (i.e., PG on the inferior parietal surface and PGm in the medial surface). These results have been repeatedly confirmed (Cavada \& Goldman-Rakic, 1989; Rozzi et al., 2006) but they are, in part, at odds with imaging data in the human brain indicating a close functional and structural link between superior and inferior parietal lobules (Caspers et al., 2011; Castiello, 2005; Lewis, 2006; Sestieri, Shulman, \& Corbetta, 2017).

More recently, the development of MRI diffusion tractography has allowed for the visualisation of long association and projection pathways of the parietal lobe in humans and comparison between species (Catani \& de Schotten, 2012; 
Thiebaut de Schotten, Dell'Acqua, Valabregue, \& Catani, 2012). While many homologies exist, especially for the frontoparietal networks (Makris et al., 2005; Thiebaut de Schotten et al., 2011), differences are also evident, for example, for those parietal areas connected to the superior colliculus (Rushworth, Behrens, \& Johansen-Berg, 2006). Tractography has also been used to cluster small intraparietal connections of the human brain. Using automatic clustering algorithms, several papers described short connections between postcentral gyrus, superior parietal lobule, and inferior parietal lobule (Guevara et al., 2011, 2017; Zhang et al., 2010). Some of these tracts are thought to have a correspondence to connections described in the monkey brain using axonal tracing studies (Caspers et al., 2011), although a direct tractographybased comparison between the two species has never been performed.

In this study, we therefore set out to explore the pattern of intraparietal lobe connectivity of the monkey and human brain using tractography-based dissection. Our first aim was to comprehensively map tracts of the human brain and propose a novel nomenclature of large bundles that could help interpret findings from functional brain imaging and clinical studies. We also directly compared tractography results obtained from human and monkey brains to identify interspecies analogies and differences. Our comparative results are discussed in light of previous tractography and axonal tracing studies and limitations of current tractography methods.

\section{Materials and methods}

\subsection{Subjects, diffusion data acquisition, and processing}

\subsubsection{Human datasets}

For the human virtual dissections, first a high quality dataset acquired from a 31-year-old healthy female volunteer was used. Peripherally cardiac-gated diffusion-weighted acquisitions were obtained on a 3T GE MR750 Discovery scanner equipped with a 32 channels Nova head-coil and with the following parameters: 90 diffusion directions, 4 non-diffusion weighted images, $2 \mathrm{~mm}$ isotropic, and $b$-value of $2000 \mathrm{sec} /$ $\mathrm{mm}^{2}$ ( $\mathrm{TE}=69 \mathrm{msec}, \mathrm{TR}=4000 \mathrm{msec} / 4 \mathrm{RR}$ intervals). Acquisition was in-plane-accelerated using an ARC factor $=2$ and slice-accelerated using a multiband factor $=3$. To increase data quality, acquisition was repeated 4 times, each time reversing the EPI phase direction. A high resolution structural SPGR-T1 weighted volume was also acquired with an isotropic resolution of $0.9 \mathrm{~mm}$ (flip angle $=12^{\circ}, \mathrm{TE}=3.2, \mathrm{TR}=8.2, \mathrm{TI}=450$ ).

Diffusion data was then corrected for subject motion, inhomogeneity distortions and eddy currents, with topup and eddy tools (https://fsl.fmrib.ox.ac.uk/fsl/) using the leastsquares resampling option (Andersson \& Sotiropoulos, 2015). The diffusion-weighted dataset was then linearly registered to the T1-weighted volume in the conformed space of Freesurfer using rigid body transformation and spline interpolation (Jenkinson, Bannister, Brady, \& Smith, 2002).

StarTrack (http//www.mr-startrack.com/) was used to generate both diffusion tensor and spherical deconvolution tractography. Diffusion tensor-based tractography was obtained using a fractional anisotropy (FA) threshold of .15, an angle threshold of $20^{\circ}$, and a step-size of $.5 \mathrm{~mm}$. Spherical deconvolution-based tractography was obtained by using the damped Richardson-Lucy algorithm with the following parameters: $\alpha=1.5 ; 200$ iterations; $\eta=.04 ; \nu=15$; abs $=.0038$ (Dell'Acqua et al., 2010; Dell'Acqua, Simmons, Williams, \& Catani, 2013). Tracking was performed using a Euler-like algorithm with a step size of $.5 \mathrm{~mm}$ and an angle threshold of $45^{\circ}$.

Results from the tractography dissection performed in the dataset described above were replicated in 20 right-handed subjects (10 males) aged 25-35 using preprocessed diffusion data $\left(b=3000 \mathrm{sec} / \mathrm{mm}^{2}\right.$, 90 directions, 18 b0 volumes and isotropic voxel size of $1.25 \mathrm{~mm}$ ) from the Human Connectome Project (http://www.humanconnectome.org). The tractography datasets for these 20 subjects used the same parameters described above.

\subsubsection{Monkey datasets}

The monkey datasets included 4 vervets (Chlorocebus aethiops), mean age $4.1 \pm 1.5$ years, 5 rhesus macaques (Macaca mulatta), mean age $11.2 \pm 2.0$ years and 2 cynomolgus macaques (Macaca fascicularis), mean age estimated to be at least 11 years. All monkeys were male and datasets were acquired on their ex vivo brains.

The vervet monkey datasets were acquired from the Behavioral Science Foundation, St. Kitts. Ethical approval for experimental procedures was provided by the Institutional Review Board of the Behavioral Science Foundation. Diffusion weighted MRI data were acquired on a $4.7 \mathrm{~T}$ Agilent Varian Inova scanner at the Danish Research Centre for Magnetic Resonance (DRCMR), Denmark. Datasets were acquired with 87 diffusion weighted directions, 16 non-diffusion weighted directions, $.5 \mathrm{~mm}$ isotropic voxel size, and a b-value of $3151 \mathrm{sec} / \mathrm{mm}^{2}$. For a full description of the tissue preparation and acquisition parameters see (Dyrby et al., 2011).

The rhesus macaque datasets were obtained from the University of Oxford. Experimental procedures were conducted in line with UK Home Office and European Union regulations (EU directive 86/609/EEC; EU Directive 2010/63/EU) Act (1986). Tissue preparation was done as described in (Large et al., 2016) and (Dyrby et al., 2011). Diffusion data were acquired using the same ex vivo MRI set up at DRCMR as above, with 61 diffusion weighted volumes, 3 non-diffusion weighted volumes, $.5 \mathrm{~mm}$ isotropic voxel size and a b-value of $4310 \mathrm{sec} / \mathrm{mm}^{2}$.

The cynomolgus macaque datasets were obtained from the Martinos Center for Biomedical Imaging and all procedures were approved by the required institutional animal care panels, as described in (de Crespigny et al, 2005). Diffusion MRI data were acquired on a 4.7T Oxford magnet with Bruker Biospec Avance console, as described in (D'Arceuil, Westmoreland, \& de Crespigny, 2007), with the following parameters: 128 diffusion weighted images, 12 non-diffusion weighted images, $.43 \mathrm{~mm}$ istotropic voxel size and a b-value of $8000 \mathrm{sec} / \mathrm{mm}^{2}$. Eight diffusion weighted volumes were removed from both datasets during quality control due to the presence of artifacts, making the total number of directions analysed 119.

All monkey datasets were scanned ex vivo using spin echo pulse sequences which are robust to image distortions. Quality checks were done visually using ExploreDTI's outlier inspection tool and no further processing was needed prior to tractography processing. 
As was done with the human data, the monkey datasets were processed with StarTrack to generate both diffusion tensor and spherical deconvolution tractography results. As spherical deconvolution-based tractography can be sensitive to noise and the ex vivo monkey datasets had varying levels of noise and voxel sizes, the parameters used for diffusion modelling and tractography were optimised individually for each dataset, and tractography datasets were evaluated by an expert anatomist (M.C.) Parameters for tractography included: $\alpha=.1-.3 ;$ 1000-3000 iterations; abs = .15-.2; angle 35-45 degrees.

\subsection{Tract dissection and visualization}

Virtual tractography dissections were performed in TrackVis (http://trackvis.org) using multiple ROIs delineated on the T1weighted images according to surface anatomical landmarks (Fig. 1).

\subsubsection{Visualization of tracts and cortical projections}

The pial and white matter surfaces of a representative human brain were calculated from T1-weighted images using the standard Freesurfer pipeline (Fischl et al., 2004). The terminal projections of the dissected tracts were displayed on the brain surface using a in house software developed in Matlab (www. mathworks.com) by $A B$ and FdSR. Briefly, all points on the white matter surface within $3 \mathrm{~mm}$ from the end-points of each streamline were selected. The white matter surface was preferred over the pial surface because of its spatial proximity to the end-points of the streamlines that, in tractography, often terminate before entering the grey matter. To account for the variability of the distance between the streamline endpoints and the white matter surface, a value of $3 \mathrm{~mm}$ was empirically chosen. This value allowed a sufficient number of streamlines to project within the gyral surface while avoiding erroneous leakage into adjacent gyri. Finally, given the 1:1 correspondence between the white and pial surfaces, the final cortical projections were obtained by selecting those pial vertices that match the white vertices previously selected. The final visualization was performed in Surf Ice (https://www. nitrc.org/projects/surfice/). A similar pipeline was used to display the cortical projections of a representative monkey brain using BrainSuite (Shattuck \& Leahy, 2002). While this method gives an approximate indication of the most likely cortical projections of the streamlines, it is important to note that streamlines' endpoints may not overlap exactly to their surface representations.

\subsection{Post-mortem human dissections}

Post-mortem white matter dissections were performed based on the Klingler method (Ludwig \& Klingler, 1956). The brain was that of a male donor who died at the age of 89 years due to pneumonia and heart failure. After removal of the dura mater

\section{A}
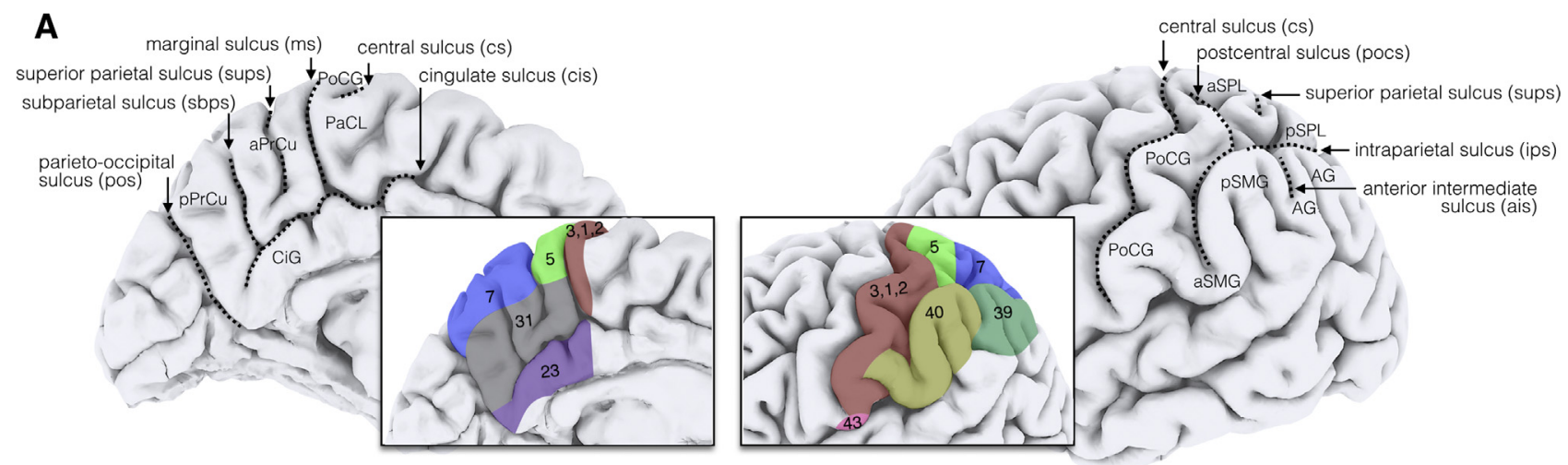

B
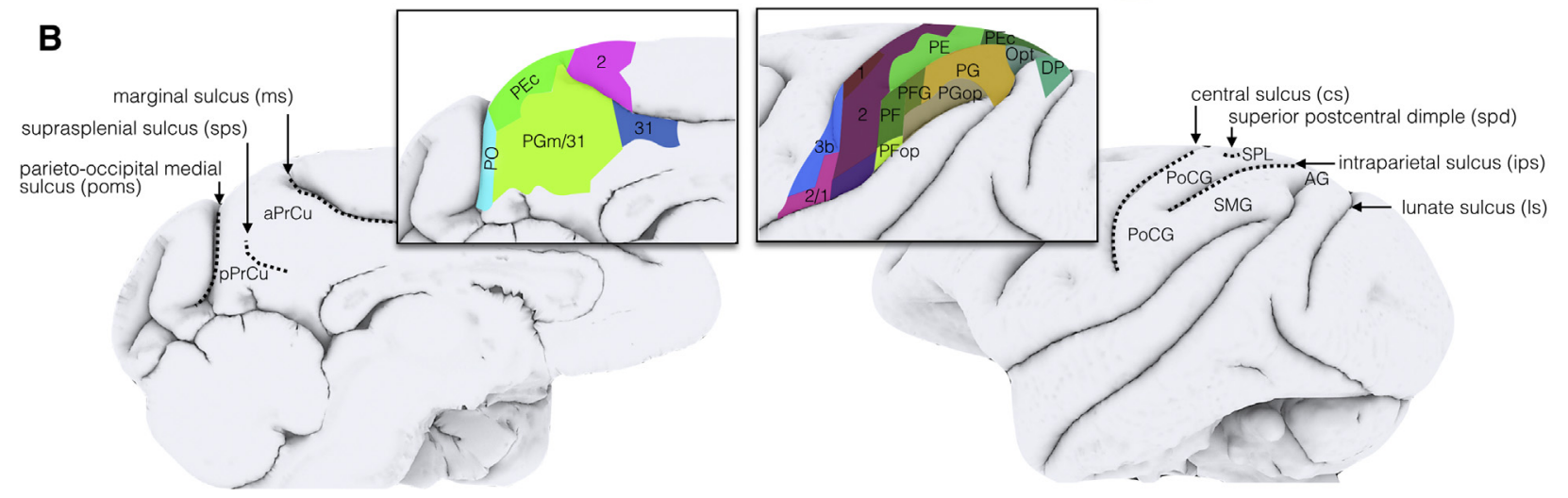

Fig. 1 - Surface anatomy and cytoarchitectonic areas of the parietal lobe for (A) human and (B) macaque brain. Regions of interest for the tractography dissections were delineated using surface landmarks and corresponded to the postcentral gyrus, the superior parietal lobule, the supramarginal gyrus and the angular gyrus. CiG, cingulate gyrus; PoCG, postcentral gyrus; PaCL, paracentral lobule; aPrCu, anterior precuneus; pPrCu, posterior precuneus; aSPL, anterior superior parietal lobule; pSPL, posterior parietal lobule; aSMG, anterior supramarginal gyrus; pSMG, posterior supramarginal gyrus; AG, angular gyrus. The simian cytoarchitectonic areas are from (Paxinos, Huang, \& Toga, 2000). 
and separation of the hemispheres, the brain was initially frozen at $-18^{\circ} \mathrm{C}$ for one week. The brain was then immersed in water and allowed to thaw at room temperature before being frozen again for eight months. Finally, the brain was defrosted before commencing the dissections. Due to the higher water content in grey matter compared to white matter, the water crystallisation process that occurs during freezing disrupts the cortex and, to a lesser extent, the underlying white matter. Thereafter, the cortex can be easily removed and the white matter peeled off to visualise bundles of fibres.

The surface anatomy of the specimen was studied carefully before dissection. The dissection was then performed by first removing the superficial cortex using a wooden spatula, slowly revealing the underlying white matter. In a stepwise fashion, these fibres were then dissected with the blunt spatula, avoiding the creation of spurious tracts. Digital pictures were taken throughout the process.

\section{Results}

Results of the tractography dissections of the short intraparietal tracts are presented according to a classification into intergyral (i.e., between postcentral gyrus, inferior parietal gyri, and superior parietal lobule) and intragyral groups (i.e., projections within a single gyrus). This classification is borrowed from a previous nomenclature applied to the frontal lobe (Catani, Dell'Acqua, Vergani et al., 2012) and recently adopted by the Terminologia Neuroanatomica (TNA), the official terminology of the IFAA (International Federation of Associations of Anatomists) (Donkelaar et al., 2017). Fig. 2 shows the anatomy of the intraparietal tracts for a representative human and monkey brain. In a previous study we indicated all frontal lobe connections with acronyms beginning with letter ' $F$ ', for example FAT for Frontal Aslant Tract (Catani, Dell'Acqua, Vergani et al., 2012). To maintain consistency, we have indicated all parietal lobe tracts with acronyms beginning with the letter ' $\mathrm{P}$ '. All tracts presented here were consistently identified in all human and monkey datasets in both hemispheres.

\subsection{Parietal Inferior-to-Superior Tract (PIST)}

This group of U-shaped tracts runs just beneath the surface of the intraparietal sulcus and connects the inferior and superior parietal lobules. In the human brain (Fig. 3, A), the PIST can be divided into an anterior component between the supramarginal gyrus (BA 40) and the superior parietal lobule
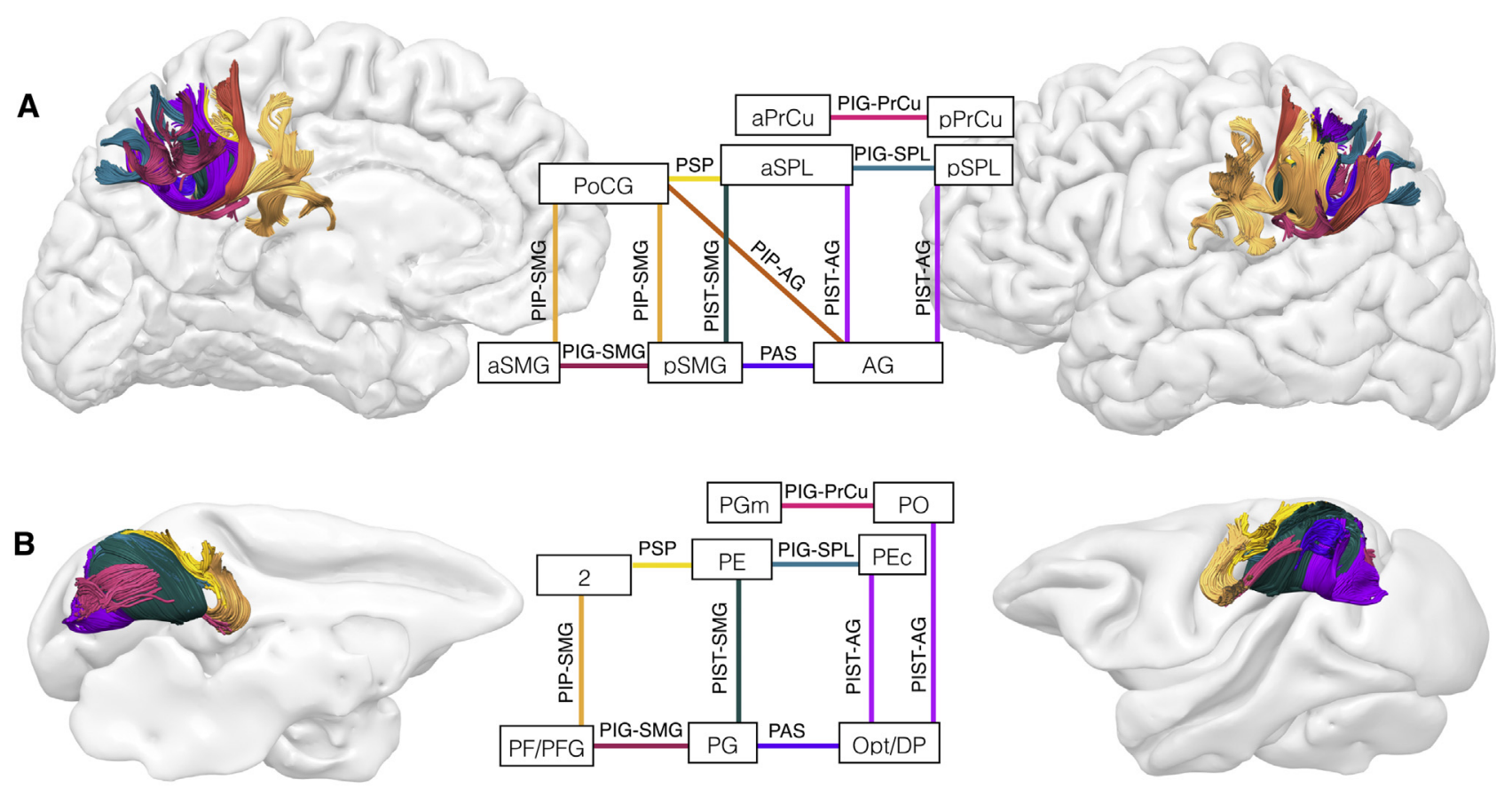

Parietal Inferior-to-Superior Tract, Supramarginal (PIST-SMG)

Parietal Inferior-to-Superior Tract, Angular (PIST-AG)

Parietal Inferior-to-Postcentral, Supramarginal (PIP-SMG)
Parietal Angular-to-Supramarginal (PAS)

Parietal Inferior-to-Postcentral, Angular (PIP-AG)

Parietal Superior-to-Postcentral (PSP)
Parietal Intragyral, Supramarginal (PIG-SMG)

Parietal Intragyral, Precuneus (PIG-PrCu)

Parietal Intragyral, superior parietal (PIG-SPL)

Fig. 2 - Tractography results of the intrinsic parietal connections in (A) human and (B) monkey brain. Left images (lateral view), right images (medial view) of the left hemisphere. The central diagrams show similarities and differences between human and monkey networks. Connections from the postcentral gyrus to the posterior supramarginal gyrus and angular gyrus were identified only in human brains. CiG, cingulate gyrus; PoCG, postcentral gyrus; PaCL, paracentral lobule; aPrCu, anterior precuneus; pPrCu, posterior precuneus; aSPL, anterior superior parietal lobule; pSPL, posterior parietal lobule; aSMG, anterior supramarginal gyrus; pSMG, posterior supramarginal gyrus; AG, angular gyrus. 

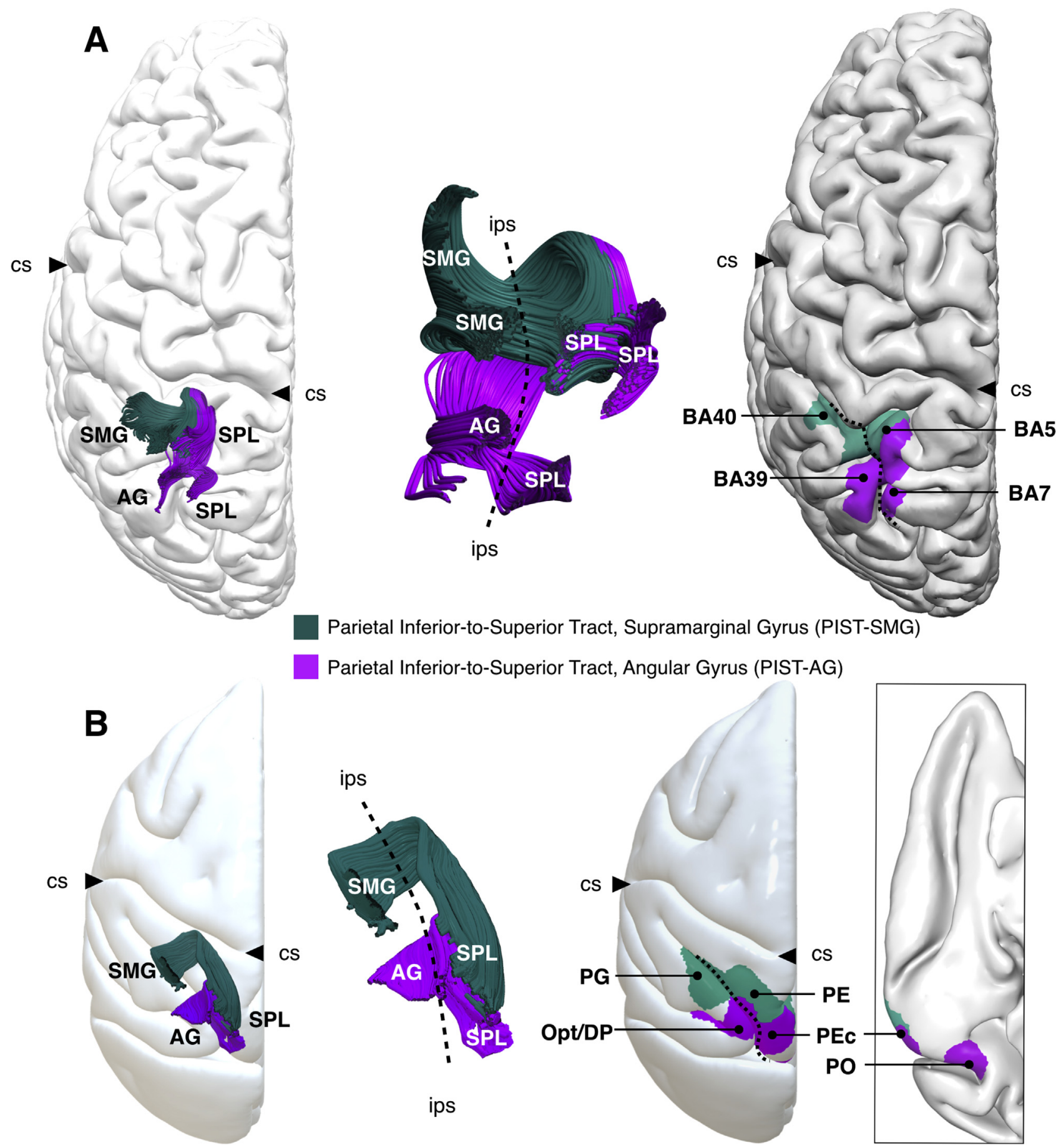

Parietal Inferior-to-Superior Tract, Supramarginal Gyrus (PIST-SMG)

Parietal Inferior-to-Superior Tract, Angular Gyrus (PIST-AG)
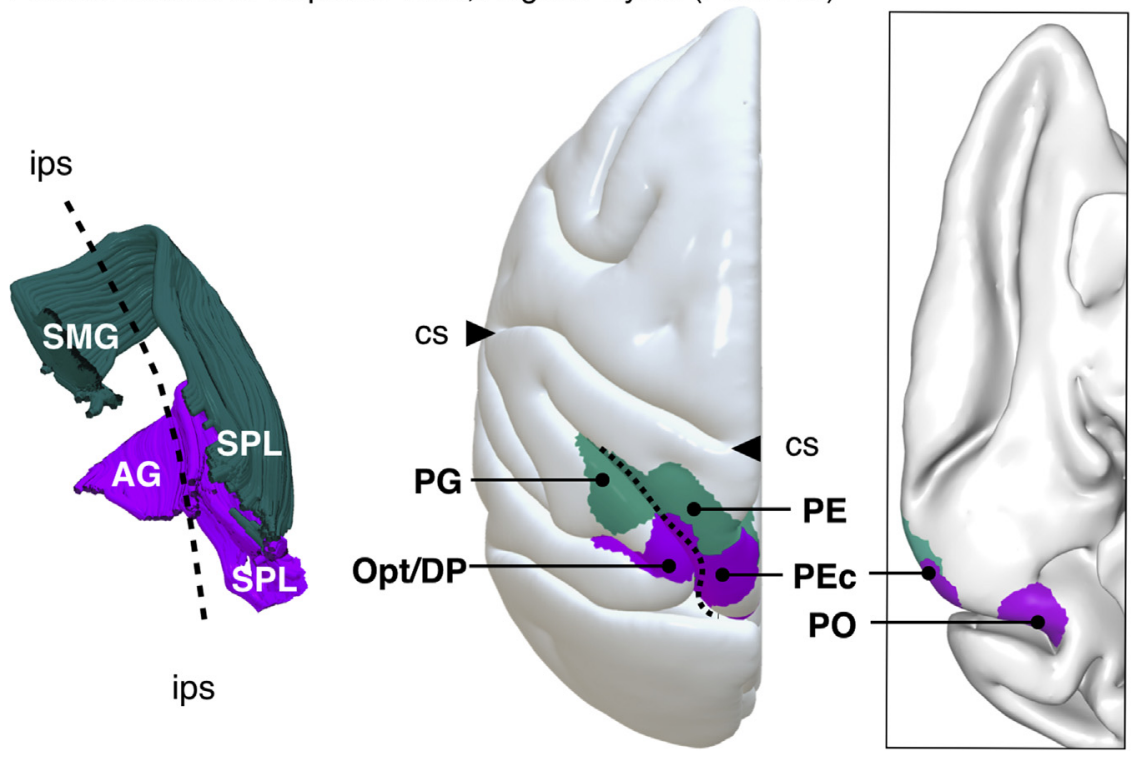

Fig. 3 - Parietal Inferior-to-Superior Tract (PIST). The inferior and superior parietal (SPL) lobules are connected by tracts running within the superficial white matter underneath the intraparietal sulcus (ips, indicated with a dashed line). The PIST is separated into an anterior and a posterior component projecting to the supramarginal (PIST-SMG) and angular (PIST-AG) gyrus, respectively. These two components are present in both (A) human and (B) monkey brains. In the monkey brain the connections of the PIST reach the medial surface of the precuneus as shown in the lower right panel; cs, central sulcus.

(mainly BA 5) (i.e., PIST-SMG), and a posterior component between the angular gyrus (BA 39) and the superior parietal lobule (both BA5 and BA7) (i.e., PIST-AG). In the monkey brain (Fig. 3, B), the PIST-SMG connects areas PE and PEc in the superior parietal lobule to area PG in the inferior parietal lobule. The PIST-AG connects PEc to area Opt and DP.

\subsection{Parietal Inferior-to-Postcentral (PIP) tract}

This is a complex group of connections in the human brain that shows significant differences between the two species. The connections between the postcentral gyrus and the supramarginal gyrus (PIP-SMG) were identified in both 
human and monkey brains, while connections between postcentral gyrus and angular gyrus (PIP-AG) only in the human brain.

In humans, the PIP is composed of short and long U-shaped tracts that extend along almost the entire surface of the postcentral gyrus regions (Fig. 4). Within the PIP-SMG the short U-shaped tracts connect the ventral postcentral gyrus to the anterior supramarginal gyrus, whereas the longest tracts connect the middle region of the postcentral gyrus to the posterior supramarginal gyrus. The PIP-AG is a smaller but longer tract compared to the PIP-SMG. It projects mainly to the hand-knob region of the postcentral gyrus and the dorsal region of the angular gyrus after passing beneath the postcentral and intraparietal sulci. The PIP-AG is always located deeper in the white matter as compared to the streamlines of the PIST-AG.

In the monkey brain, the PIP-SMG is consistently observed as a set of vertical tracts connecting the postcentral gyrus to areas PF and PFG in the supramarginal gyrus (Fig. 5). The PIPSMG in the monkey brain passes under the anterior segment of the intraparietal sulcus. Connections between postcentral and angular gyrus equivalent to the human PIP-AG were not identified in the monkey brain.

\subsection{Parietal Superior-to-Postcentral (PSP)}

This bundle connects the most dorsal region of the postcentral gyrus to the superior parietal lobule. In the human

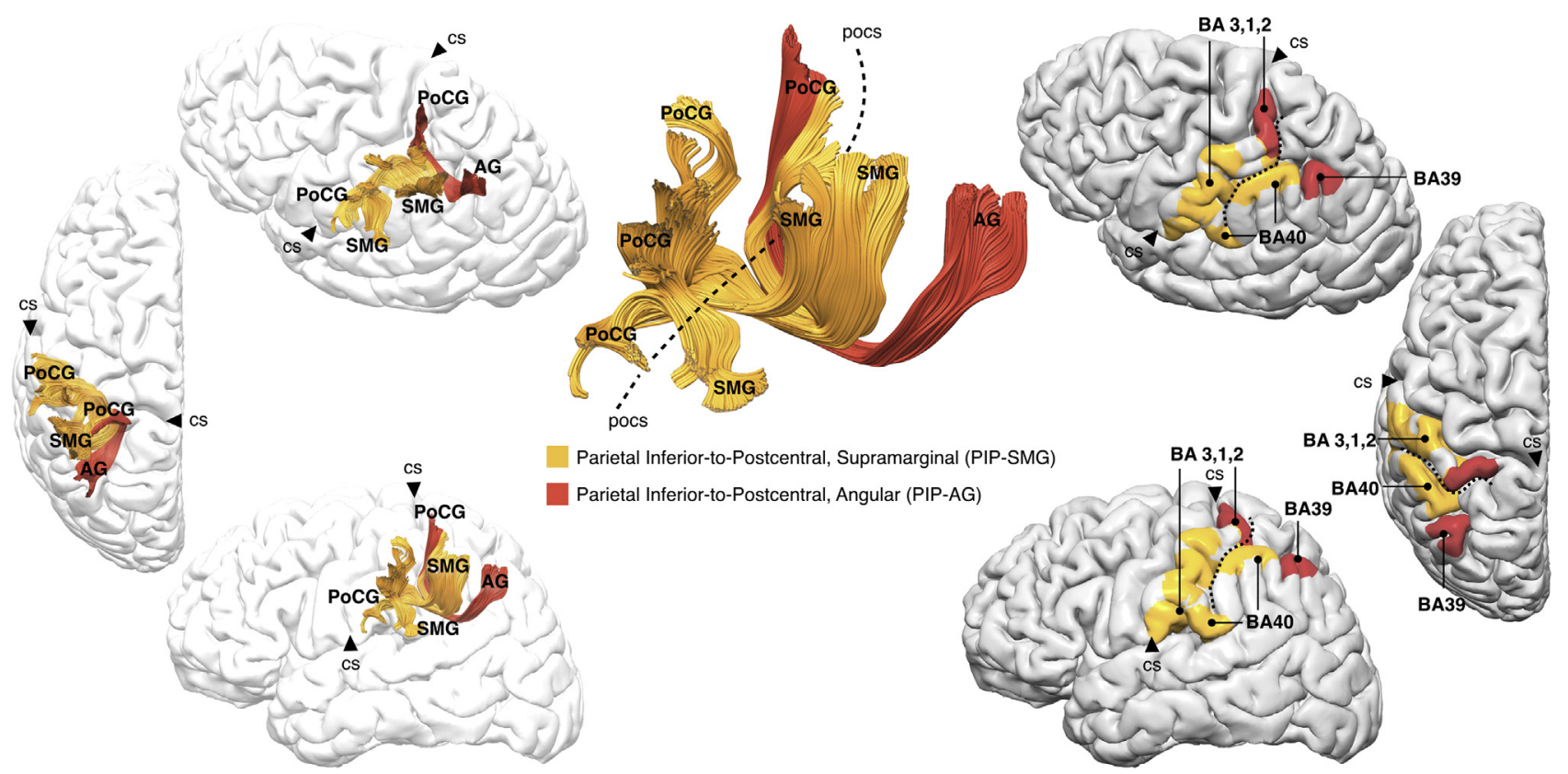

Fig. 4 - Parietal Inferior-to-Postcentral (PIP) tract in the human brain. The streamlines of the PIP can be grouped into an anterior and a posterior component projecting to the supramarginal (PIP-SMG) and angular (PIP-AG) gyrus, respectively. The postcentral sulcus (pocs) is indicated with a dashed line; cs, central sulcus.

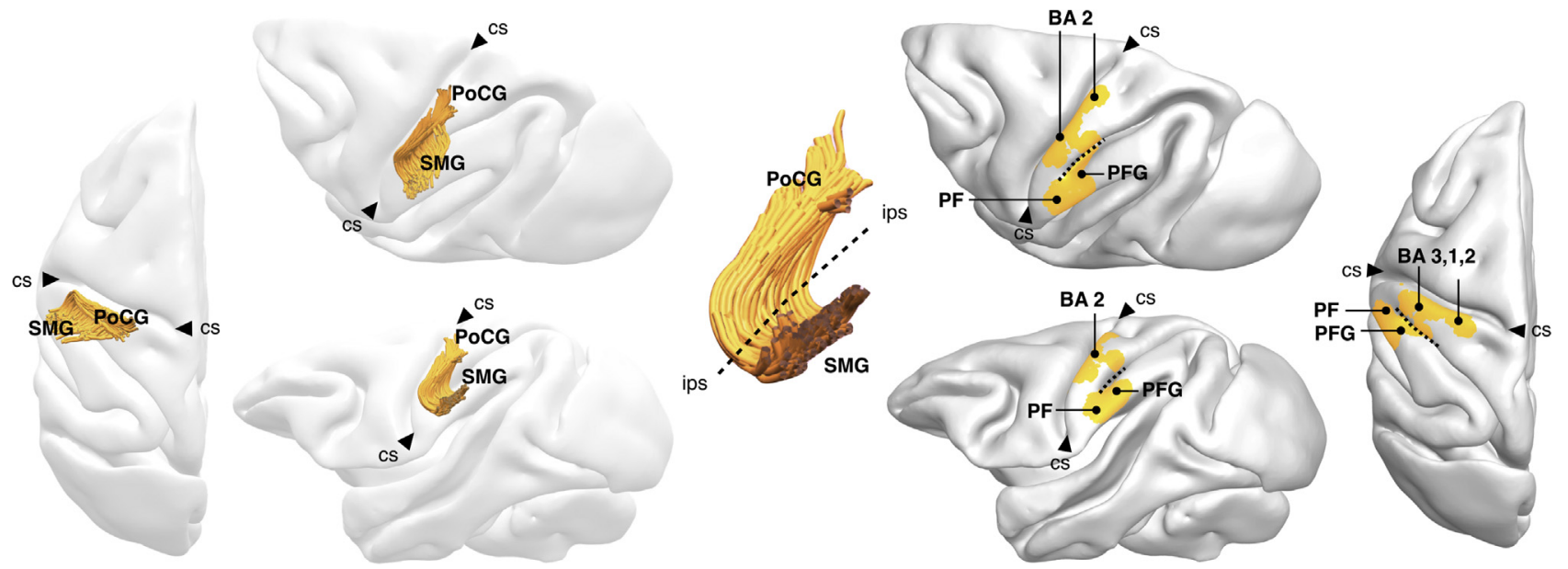

Fig. 5 - Parietal Inferior-to-Postcentral (PIP) tract in the monkey brain. This tract connects the anterior areas of the supramarginal gyrus (PIP-SMG) to area 2 of the postcentral gyrus. The intraparietal sulcus (ips) is indicated by a dashed line; cs, central sulcus. 
brain the PSP is composed primarily of short U-shaped tracts travelling underneath the postcentral sulcus (Fig. 6, A). There is a high degree of variability in the anatomy of this tract in the human brain. In the monkey brain (Fig. 6, B), the PSP extends more posteriorly and projects to both $\mathrm{PE}$ and PEc in the superior parietal lobule.

\subsection{Parietal angular-to-supramarginal (PAS)}

This is a small U-shaped tract between the anterior regions of the angular gyrus and the posterior supramarginal gyrus. In humans, the PAS is oriented with its concavity facing the dorsolateral surface of the parietal lobe and courses around the anterior intermediate sulcus, which separates the angular from the supramarginal gyrus (Fig. 7, A). In the monkey, brain the PAS runs medially and dorsally to the tip of the superior temporal sulcus and connects areas DP, Opt and posterior PG (Fig. 7, B).

\subsection{Parietal Intra-Gyral (PIG) tracts}

Several short tracts connecting different regions within the same gyrus can be consistently identified in both human and monkey brains. In general, in the human brain these tracts are U-shaped whereas in the monkey brain they show more linear trajectories.
3.5.1. PIG of the supramarginal gyrus (PIG-SMG)

In the human brain, this tract connects anterior and posterior regions of the supramarginal gyrus (Fig. 7, A). In monkeys, it allows communication between PF, PFG and PG (Fig. 7, B).

\subsubsection{PIG of the precuneus (PIG-PrCu)}

In the human brain, this tract is composed of a series of short U-shaped tracts connecting anterior, intermediate, and posterior regions of the precuneus (Fig. 8, A left). Their shape is determined by the presence of the subparietal sulcus; when the latter is deep the fibres are U-shaped, when shallow the fibres show a more longitudinal and straight course. In the monkey brain, they connect PO to PGm/31 and compared to humans traverse a more longitudinal course due to the lack of depth of the suprasplenial sulcus (Fig. 8, B left).

\subsubsection{PIG of the superior parietal lobule (PIG-SPL)}

In the human brain, several short U-shaped tracts connecting dorsal regions of BA 5 and BA 7 were identified (Fig. 8, A right). In the monkey brain, only a single bundle could be tracked, running between $\mathrm{PE}$ and PEc (Fig. 8, B right).

\subsection{Negative tractography findings}

In our study we were able to identify connections between lateral inferior parietal lobule (PG, Opt) and areas PEc and PO of

A
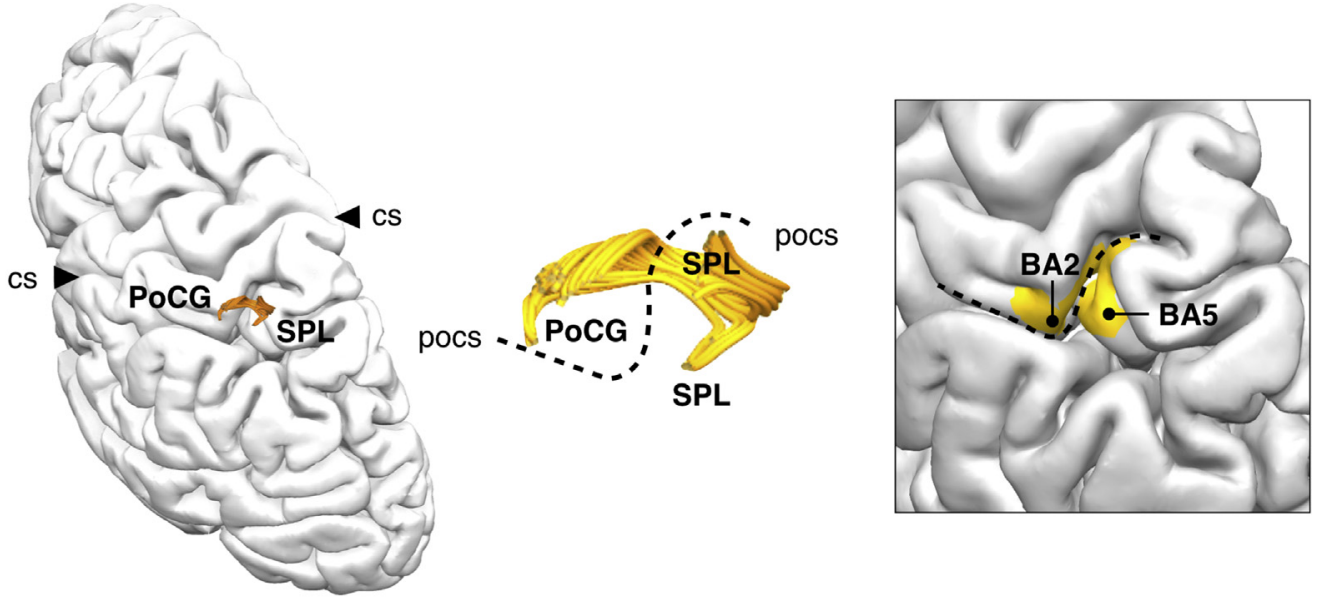

B
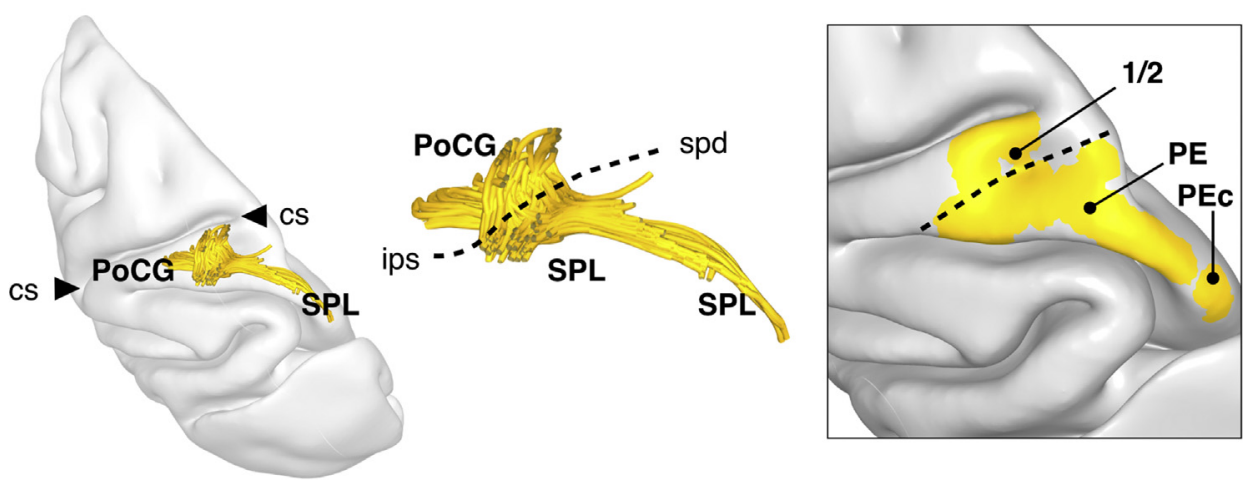

Fig. 6 - Postcentral-to-Superior Parietal (PSP) tract connecting the postcentral gyrus (PoCG) and the superior parietal lobule (SPL). (A) In the human brain, the PSP is a U-shaped tract running underneath the postcentral sulcus (pocs, indicated by a dashed line). (B) In most of the monkey brains used for this study, the pocs is not present and the PSP crosses a line between the superior posterior dimple (spd) and the intraparietal sulcus (ips); cs, central sulcus. 


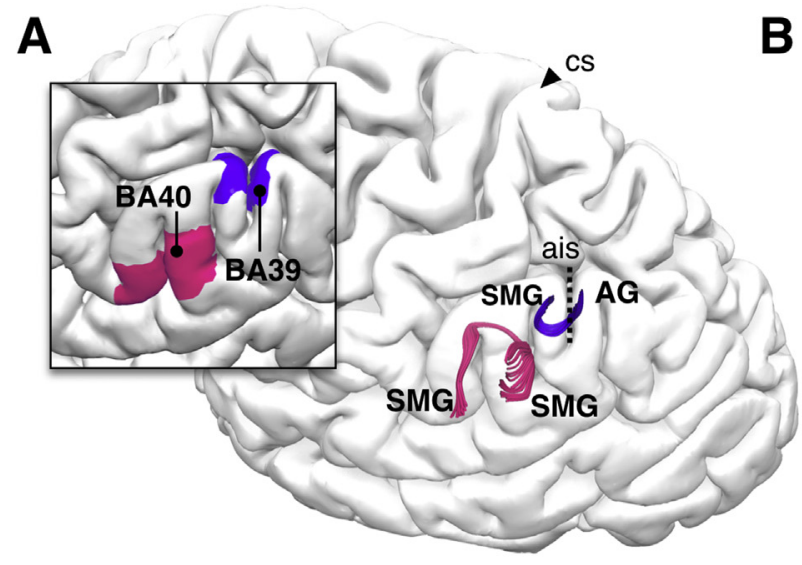

B

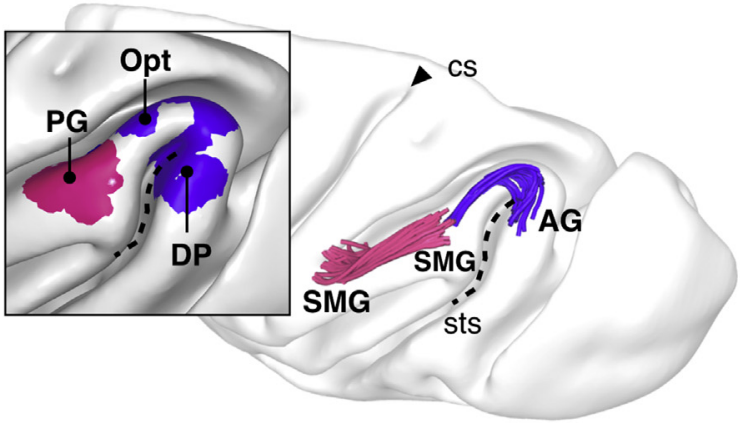

Parietal Angular to Supramarginal Gyrus (PAS)

Parietal Intra-Gyral, Supramarginal Gyrus (PIG-SMG)

Fig. 7 - Connections between regions of the inferior parietal lobule. (A) In the human brain the parietal angular-tosupramarginal (PAS) tract is located underneath the anterior intermediate sulcus (ais, indicated by a dashed line). The Parietal Intra-Gyral tract of the supramarginal gyrus (PIG-SMG) is often located around the ascending terminal branch of the sylvian sulcus (sbsf). (B) In the monkey brain, the PAS arches around the posterior tip of the superior temporal sulcus (sts, indicated by a dashed line) and connects posterior PG, Opt and DP. The PIG-SMG runs between anterior (PF), intermediate (PFG), and posterior (PG) areas of the supramarginal gyrus. Only the PG area is indicated in the figure; cs, central sulcus.

the precuneus but only in the monkey brains (Fig. 3B). Connections between the inferior parietal lobule and area PGm have been consistently reported when using axonal tracers in monkeys (Pandya \& Seltzer, 1982; Rozzi et al., 2006) but we were unable to visualise them with tractography in either species.

\subsection{Post-mortem dissections}

All tracts described with tractography were visible on the post-mortem brain using the Klinger's method (Fig. 9). Most of these tracts run on the superficial layers of the white matter except for the PIP-AG, which is deeper than the others. This tract was followed until its intersection with other projections tracts, after which it was difficult to separate it from the other white matter tracts.

\subsection{Discussion}

In this study, we propose a nomenclature for short association fibres of the parietal lobe based on the description of large pathways identified with tractography. All proposed tracts were identified both in human and monkey datasets and compared with previous data from axonal tracing studies. Interspecies differences were evident for some tracts and these will be discussed in light of the previous literature and functional considerations. Hereafter the anatomy and possible functional and clinical correlates of the principal tracts are discussed, followed by a description of the general principles of white matter organization of the intralobar parietal networks.

3.8.1. Tracts of the intraparietal sulcus connecting superior and inferior parietal lobules (PIST)

Our tractography findings in the monkey brain indicate the presence of connections between the superior parietal lobule
(PE, PEc) and the most posterior regions of the inferior parietal lobule (DP, Opt, PG). On the medial surface these projections reach the medial $\mathrm{PEC}$ and $\mathrm{PO}$. This connectivity pattern corresponds to the results of a recently published axonal tracing study in macaque monkeys (Rozzi et al., 2006). We found a similar organization in the human brain except for the absence of the medial projections to the precuneus. This may be related to the limitations of current tractography methods in visualizing crossing or merging tracts (Dell'Acqua \& Catani, 2012). Alternatively, the human connectivity between the inferior parietal cortex and the precuneus may be more complex and polysynaptic - mediated by two or more tracts due to the expansion of some areas of the superior parietal lobule (Scheperjans et al., 2008).

Functional considerations regarding the role of the PIST can be indirectly inferred from its cortical projections. This tract connects not only superior and inferior parietal cortex but also different areas within the intraparietal sulcus. These areas, which are subdivided into anterior, medial, lateral and caudal intraparietal sections (AIP, MIP, LIP, CIP) may serve similar functions across species (Borra \& Luppino, 2017; Grefkes \& Fink, 2005). They have been shown to be involved in saccadic eye movement, object identification and orientation (both tactile and visual), and reaching and grasping. The function of the PIST may, therefore, relate to complex tasks requiring integration of visual and somatosensory information for object identification and online updating of reaching and grasping movements (Grefkes \& Fink, 2005).

While these areas of the intraparietal sulcus can be easily accessed in the monkey brain for experimental studies, in humans it is rather difficult to perform functional imaging or lesion studies that show a clear separation of individual areas due to the close proximity of the upper and lower bank of the intraparietal cortex and the low spatial resolution of current fMRI. Nevertheless, irrespective to the details of the exact cortical areas a clear dissociation between lesions of the 

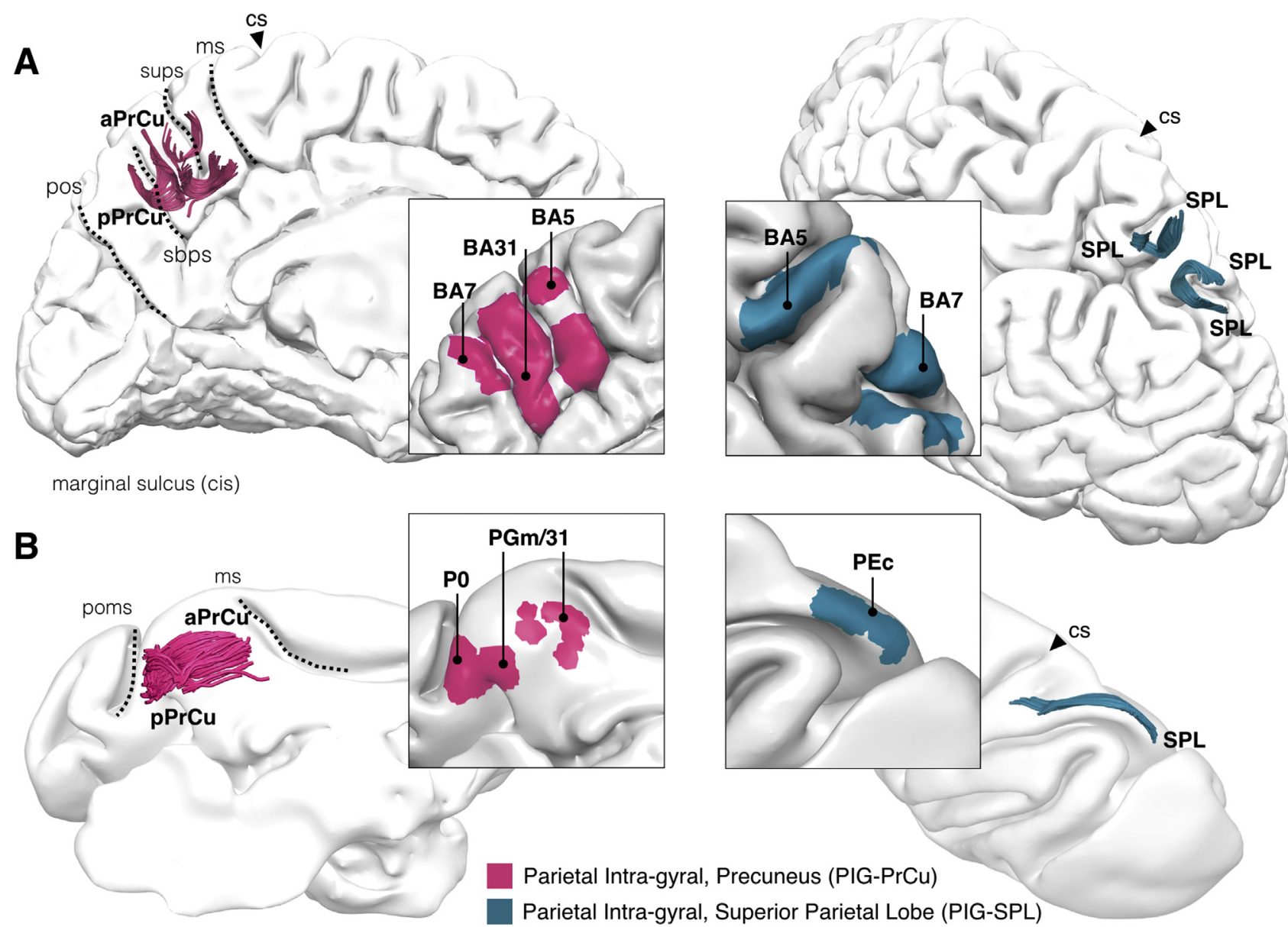

Fig. 8 - Parietal Intra-Gyral connections of the precuneus (PIG-PrCu) and superior parietal lobule (PIG-SPL). (A) In the human brain PIG-PrCu is a complex group of tracts running between the marginal sulcus and the parieto-occipital sulcus. Their anatomy varies according to the morphology of the superior parietal sulcus (sups) and subparietal sulcus (sbps). Within the superior parietal lobule, PIG-SPL connections link anterior to posterior areas and medial to lateral areas. (B) In the monkey, the PIG-PrCu runs between the parieto-occipital medial sulcus (poms) and the marginal sulcus (ms) connecting area PO to area PGm/31. The PIG-SPL is a single tract with longitudinal course between anterior (PE, not shown in the figure) and posterior (PEc) areas of the superior parietal lobule.

superior and inferior parietal lobule has been observed in human lesion studies (Catani, Dell'Acqua, Bizzi et al., 2012; Critchley, 1953; Jeannerod, 2006; Mountcastle, 1995; Rizzolatti \& Matelli, 2003). Lesions of the posterior regions of the superior parietal lobule can manifest with optic ataxia, a condition characterized by the inability to perform spatially accurate movements towards visual targets (Karnath \& Perenin, 2005; Martin, Karnath, \& Himmelbach, 2015; Pisella, Binkofski, Lasek, Toni, \& Rossetti, 2006). In these patients, the deficit is particularly evident for peripherally located objects, for which a correct reach-to-grasp action requires coordinated saccade movements for a dynamic update of visual target location (Caminiti et al., 2010; Grafton, 2010). Lesions to the inferior parietal lobule can present with apraxia, a deficit of action control for tool use, gesture imitations or assembling objects (Goldenberg, 2013). These clinical data combined with experimental research in animals have suggested a dichotomy between a dorsal fronto-parietal network for control of actions "online" (reach-to-grasp pathway) and a ventral fronto-parietal network for action organization, space perception and action understanding (grasping pathway) (Rizzolatti \& Matelli, 2003). While the dichotomy is largely correct, there is also significant evidence for an interaction between the two streams. For example, the finding that reaching neurons in the superior parietal lobule can also be modulated by precision grasping movements (Fattori et al., 2009) suggests afferent projections from inferior to superior parietal regions. There is also evidence that commands related to reaching movements and involving proximal muscles modulate the activity of distal muscles involved in grasping (Davare, Kraskov, Rothwell, \& Lemon, 2011; Dominici, Popa, Ginanneschi, Mazzocchio, \& Rossi, 2005). In a recent paper Wood, Chouinard, Major, and Goodale (2017) showed that patients with posterior parietal lesions lack access to visual information about object orientation for the control of reach-to-grasp movements. We suggest that lesions to the PIST-AG may prevent the transfer of information about object visual orientation to the superior parietal lobule necessary to compute prediction of biomechanical cost and selection of most efficient behaviour. 


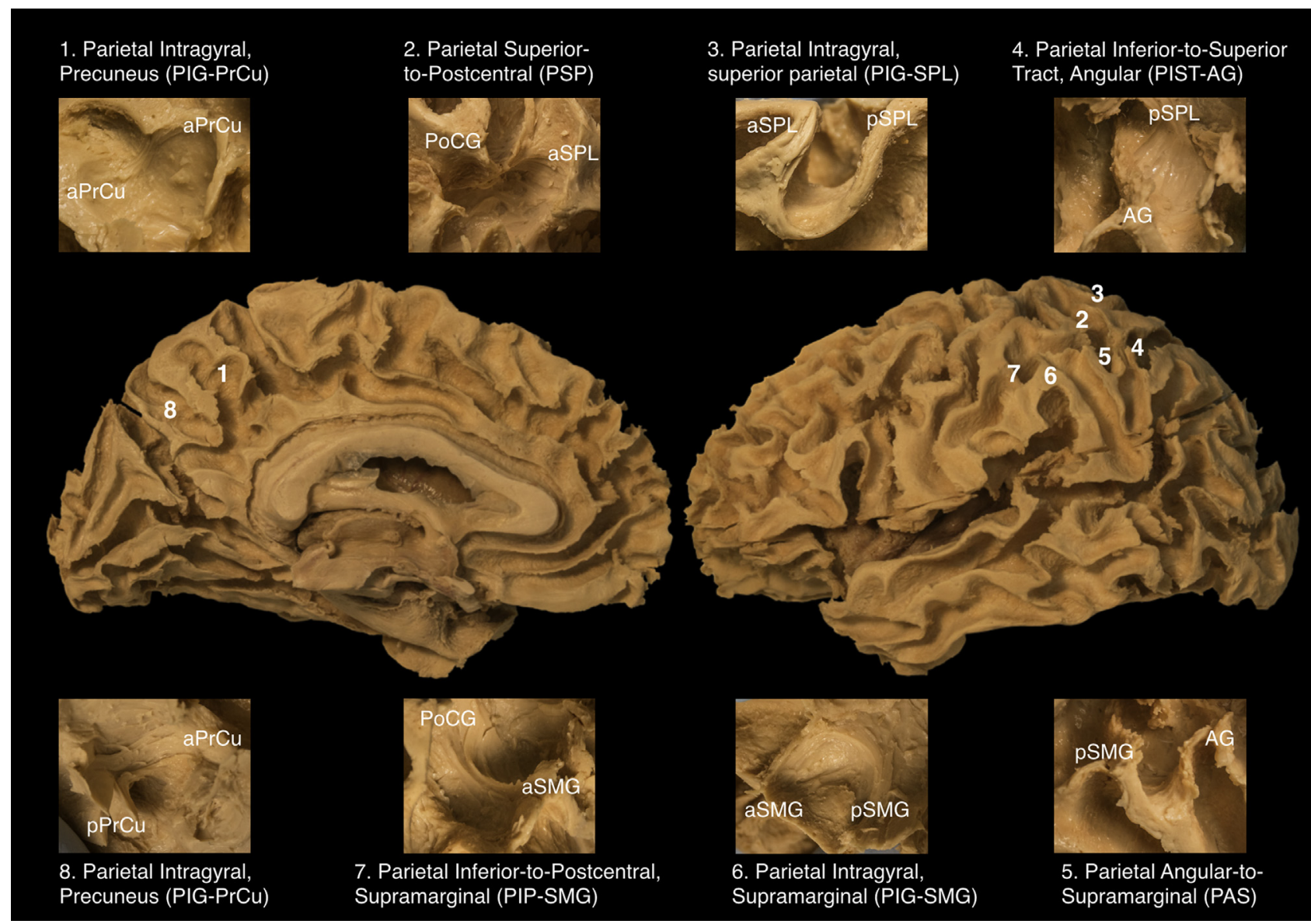

Fig. 9 - Intraparietal connections identified with post-mortem blunt dissections on the medial (left) and lateral (right) aspect of the parietal lobe. All tracts were identified on the white matter layers just beneath the cortex, except for the PIP-AG, which was located deeply in the white matter and was difficult to follow as a single continuous bundle.

Evidence for an interaction between inferior and superior parietal lobules emerges also from functional imaging studies of networks related to use of tools (Lewis, 2006) or pantomime imitation (Goldenberg, 2013). While the clinical literature points to the inferior parietal lobule (in particular supramarginal gyrus) as the key lesion site for apraxia (Martin et al., 2016), functional imaging studies show a strong activation of both superior and inferior parietal cortex for tasks involving tool use (Lewis, 2006). The involvement of the two parietal lobules in these tasks is however different. The superior parietal lobule is thought to code the location of the limbs relative to other body parts to represent one's body schema as it dynamically changes during movement. The superior parietal lobe is also more frequently lesioned in patients with impaired ability to imitate gestures. Conversely, the inferior parietal lobule, mostly the supramarginal gyrus, is activated in tasks requiring making judgements about the manipulability of objects and it appears to prepare and trigger appropriate object-related action schemas (Lewis, 2006; Verhagen, Dijkerman, Grol, \& Toni, 2008). It can be argued that motor control of object-related actions requires an interaction between the superior and inferior parietal lobules for an online control of the upper limb movements during object manipulation.

A similar cross-talk between superior and inferior parietal lobules can be argued for dorsal and ventral visuospatial attention networks. Corbetta and Shulman (2002) suggested the existence of a ventral visuospatial attentional network (VAN) for stimulus-grabbed attention and a dorsal visuospatial attentional network (DAN) that controls goal-directed attention. Although the two networks are anatomically and functionally distinct (Parlatini et al., 2017; Thiebaut de Schotten et al., 2011), goal-directed and stimulus-grabbed attention processing operates simultaneously in many tasks of everyday life. Their interaction is dynamic and task dependent, leading to either anticorrelated activity or coactivation of their parietal areas (Corbetta \& Shulman, 2011; Vossel, Geng, \& Fink, 2014). We suggest that the PIST may represent the anatomical circuit that coordinates the activity of the DAN and VAN parietal areas. This hypothesis is supported by a strong functional connectivity between superior and inferior parietal regions (Wen, Yao, Liu, \& Ding, 2012). The PIST may mediate the mutual interaction of the parietal areas whenever one network overrides the activity of the other or both networks work conjunctly. This can occur in situations where the DAN maintains goal-directed attention despite the occurrence of unexpected stimulus, or when an unexpected stimulus determines a shift of attention of the DAN from one goal to another. These functional interactions have been studied with fMRI using visual search and attentional reorienting paradigms. In attentional reorienting tasks, both 
dorsal and ventral parietal areas are activated, whereas in visual search tasks, the superior parietal lobule activates while the inferior parietal lobule deactivates. The latter pattern of activation has been interpreted as a top-down filtering mechanism that prevents irrelevant distractors disrupting goal-driven behaviour (Shulman, Astafiev, McAvoy, d'Avossa, \& Corbetta, 2007). However, deactivation of the inferior parietal regions changes into activation in visual search tasks when nontarget stimuli carry information relevant to the target stimulus (Geng \& Mangun, 2011).

These findings are particular relevant to help interpret visuospatial neglect symptoms in patients with parietal lobe lesion (Umarova, 2017). These patients fail to explore and reorient their attention to the contralesional space, suggesting an impairment of the DAN. However, most of the lesions causing visuospatial neglect are within the inferior parietal cortex and its underlying white matter connections. The anatomical link between the superior and inferior parietal lobules suggests that in neglect patients a disconnection of the PIST-AG may prevent salient information from modulating the activity of the dorsal parietal areas. This intraparietal disconnection mechanism is supported by those case reports in which neglect is caused by selective lesions of the intraparietal sulcus and underlying white matter (Gillebert et al., 2011). Disconnection of the PIST may also explain an intraparietal diaschisis mechanism resulting in the reduced activation of an intact superior parietal lobule in neglect patients with inferior parietal stroke (Corbetta, Kincade, Lewis, Snyder, \& Sapir, 2005).

In fMRI studies, the inferior parietal lobule shows also activation for episodic memory tasks (Cabeza, Ciaramelli, Olson, \& Moscovitch, 2008; Sestieri et al., 2017), working memory, and 3D object representation (Tsutsui et al., 2003; Grefkes \& Fink, 2005) whereas the superior parietal lobule is involved in movement detection, mental rotation, and visual imagery (Lewis, 2006; de Gelder, Tamietto, Pegna, \& Van den Stock, 2015). A direct link between superior and inferior parietal lobules could therefore be important in facilitating online mental manipulation of retrieved memories or visual information in tasks requiring motion and rotation of 3D objects. This hypothesis is supported by functional neuroimaging (Zacks, 2008) and lesion studies (Glass, Krueger, Solomon, Raymont, \& Grafman, 2013) reporting an association between mental rotation and activation (or lesion) of the intraparietal sulcus. In healthy subjects a positive correlation between mental rotation abilities and fractional anisotropy of superficial white matter fibres of the intraparietal sulcus has been reported (Wolbers, Schoell, \& Büchel, 2006). Finally, a possible role of the PIST in working memory (inferior parietal lobule) and visual search (superior parietal lobule) is suggested by the tendency of neglect patients to reselect previously cancelled targets (Husain et al., 2001). This indicates that the effect of working memory on visual search behaviour may be mediated by the PIST.

Although connections between inferior and superior parietal lobule are present in both human and monkey brains, this should not imply a functional equivalence between species. For example, differences between human and monkey in the functional anatomy of the VAN suggest a different role for the PIST in the simian attentional networks (Patel et al., 2015).
Future comparative studies may also reveal quantitative differences within the PIST system possibly related to the involvement of the angular gyrus in uniquely human abilities, such as writing. Current anatomical models of handwriting identify two separate but integrated networks (Planton, Jucla, Roux, \& Démonet, 2013; Purcell, Turkeltaub, Eden, \& Rapp, 2011): a central 'language' network corresponding to the arcuate fasciculus and a peripheral 'graphomotor' network, which recruits widespread dorsal fronto-parietal regions and may correspond to the dorsal branch of the superior longitudinal fasciculus (SLF I). The PIST-AG may be involved in writing through the transferal of orthographic knowledge from the angular gyrus to the superior parietal lobule. Indirect evidence for this role comes from previous lesion studies reporting dysgraphia with posterior parietal lesions (Magrassi, Bongetta, Bianchini, Berardesca, \& Arienta, 2010; Rapp, Purcell, Hillis, Capasso, \& Miceli, 2016). In these patients, the orthographic knowledge remains intact but the ability to transform it into written words is impaired, which may be due to a lesion of the PIST-angular.

\subsubsection{Tracts between the inferior parietal lobule and the postcentral gyrus (PIP)}

In our tractography results of the monkey brain, tracts connecting the postcentral regions of the hand and mouth to the inferior parietal lobule project exclusively to areas PF and PFG. This connectivity pattern has been previously documented in the monkey brain with axonal tracing methods (Pandya \& Seltzer, 1982; Rozzi et al., 2006; Schmahmann \& Pandya, 2006). These fibres appear as a compact U-shaped bundle running within the most superficial white matter layer beneath the intraparietal sulcus. This tract conveys to areas PF and PFG the tactile and proprioceptive information necessary to guide motor actions of the face and mouth, and hand/arm, respectively (Rozzi et al., 2006). This information is particularly important for the execution of precision grasping as demonstrated by experimental studies in animals (Hikosaka, Tanaka, Sakamoto, \& Iwamura, 1985) and functional neuroimaging in humans (Castiello, 2005). In addition, the anatomical proximity and cortical overlap of the projections from different body parts suggest a role in conveying tactile and proprioceptive inputs for complex behaviour requiring the simultaneous control of both hand and mouth (e.g., feeding) (Bruner et al., 2014). Lesions to the PIP may prevent somatosensory information from reaching inferior parietal lobule areas and result in disrupted selection of grasping actions observed in experimental lesion studies in monkeys (Borra \& Luppino, 2017) and in patients with apraxia (Watson \& Buxbaum, 2015).

In human functional imaging studies, the supramarginal and postcentral gyrus are simultaneously activated in tasks involving conscious perception of fearful bodies (Tamietto et al., 2015). The PIP connections may therefore allow for an enrichment of these visual experiences with somatosensory information whenever visual stimuli are consciously perceived within the frontal-parietal network (Engelen, de Graaf, Sack, \& de Gelder, 2015). Parietal lesions to the PIP may disconnect the somatosensory areas from the inferior parietal lobule and prevent a mutual exchange of information between these two regions that may underlie conditions such as anosognosia (Besharati et al., 2016). One can argue that in 
these patients, the disconnection of the PIP may prevent tactile and proprioceptive stimuli relayed in the postcentral gyrus from reaching the inferior parietal lobule for a conscious perception of the contralateral hemibody.

Our tractography reconstruction of this pathway indicates a similar gradient of spatial organization across species, from mouth/face to hand/arm (Rozzi, Ferrari, Bonini, Rizzolatti, \& Fogassi, 2008). However, in humans but not in monkeys, the most posterior PIP connections reach the posterior supramarginal gyrus and angular gyrus. The complete absence of these posterior projections in the monkey brain (Rozzi et al., 2006) suggests that this component may serve uniquely human functions. As reviewed above, damage to the left inferior parietal lobule in humans is commonly associated with apraxia. This syndrome manifests in different modalities (e.g., imitation of hand posture, use of tools, draw or build objects, etc.) (Goldenberg, 2013), some of which have not been described in experimental animal studies (e.g., constructional apraxia) (Caminiti et al., 2010). The interspecies similarities in PIP-SMG anatomy may explain why certain motor deficits that result from lesions to the anterior region of the inferior parietal lobule are observed in both monkey and humans, such as impaired hand shaping and object manipulation, which are characteristically observed in certain forms of apraxia. On the other hand, the interspecies divergence in the anatomy of the PIP-AG may explain uniquely human apraxia syndromes .

This tract may also be important for other human functions that involve tactile perception, language and communication in general. In studies looking at Braille reading, for example, activations are consistently found in the hand knob area of the post-central gyrus and inferior parietal lobule (Burton et al., 2002; Sadato et al., 1998) suggesting that this tract could represent a direct route from tactile perception to orthographic representation of words. A similar role could be attributed to these connections in the early stages of mathematical (Butterworth, 1999; Dehaene, Piazza, Pinel, \& Cohen, 2003) and quantitative estimate learning (Lecce, Walsh, Didino, \& Cappelletti, 2015).

\subsubsection{Connections between the superior parietal lobule and} the postcentral gyrus (PSP)

Axonal tracing studies have shown that direct connections exist between the postcentral gyrus (e.g., area 2) and superior parietal regions (PE) in the monkey brain (Pandya \& Seltzer, 1982). These tracts convey somatosensory information for limb position and object representation to associative somatosensory areas (Castiello, 2005; Grafton, 2010). In our tractography reconstructions, connections from the postcentral gyrus to superior parietal lobule originate primarily from the upper two thirds of the postcentral cortex, which receives proprioceptive and tactile information from the fingers and upper and lower limbs but not the mouth or tongue. This is consistent with monkey anatomical (Sakata et al., 1973) and electrophysiological data showing that area $\mathrm{PE}$ in the superior parietal lobule contains neurons with receptive fields for the shoulder, forelimb and digits but no apparent representation of other body parts (Seelke et al., 2012). Somatic receptors in muscles, joints and skin provide information regarding the current posture of the hand/arm and their location and orientation with respect to potential targets for grasping. So, the PSP, like the PIP, seems to convey somatosensory information necessary to compute a trajectory to bring the hand to the object and grasp it properly. However, the neurons in the anterior regions of the superior parietal lobule do not respond to visual stimuli, suggesting a different processing of this somatosensory information conveyed by the PSP to the superior parietal as compared to the information travelling through the PIP to the inferior parietal regions. The anatomical location of these tracts is consistent with both experimental and clinical data from subjects with brain lesions resulting in tactile astereognosia. This syndrome is characterized by impaired tactile discrimination of objects, which is severe for lesions affecting the cortical projections of the PSP to the anterior intraparietal area (AIP) (Moffett, Ettlinger, Morton, \& Piercy, 1967), which has been shown to participate in hand shaping and grasping in both monkeys and humans (Castiello, 2005; Davare et al., 2011).

Finally, the PSP is important to convey necessary somatosensory information to those regions in the anterior part of the superior parietal lobule that have a role in reconstructing a coherent body image. An abnormal development of the PSP connections may underlie xenomelia, a rare condition characterised by the persistent and compulsive desire for the amputation of one or more physically healthy limbs, which involves the lower limb in more than $80 \%$ of the cases (Hänggi et al., 2017; Hilti et al., 2012).

\subsubsection{Parietal Angular to Supramarginal (PAS) and parietal} intragyral (PIG) connections

These short fibres connecting adjacent gyri within the same lobule (e.g., PAS) or adjacent regions within the same gyrus (PIG) have been extensively documented in monkey brains using axonal tracing studies (Rozzi et al., 2006; Schmahmann \& Pandya, 2006). Tractography studies have also reported similar connections in humans (Caspers et al., 2011; Guevara et al., 2011) but their functional role remains unknown. The use of more sophisticated manipulations of functional imaging paradigms (Tyler, Dasgupta, Agosta, Battelli, \& Grossman, 2015; de Gelder et al., 2015) and voxel lesion approaches (Martin, Karnath, \& Himmelbach, 2015) is revealing a more fine-grained functional specialisation of parietal regions that have been traditionally lumped together. One possible role of these short fibres is to maintain a functional link between areas that show specialization within the same broad functional domain. This may apply to PIG connections of the posterior superior parietal lobule between areas dedicated to reaching towards preferentially peripheral targets and areas without peripheral bias (Martin, Karnath, \& Himmelbach, 2015) or to anterior and posterior precuneal regions dedicated to visual perception and imagery of angry faces (de Gelder et al., 2015). On the lateral parietal cortex, a similar role can be hypothesised for the PAS tract connecting angular to supramarginal gyrus, which may have a different role in a number of functions including tool use (Watson \& Buxbaum, 2015), visuospatial attention (Tyler et al., 2015) and speech (Tian, Zarate, \& Poeppel, 2016).

3.8.5. General organization of the intrinsic parietal networks Our tractography reconstructions of the intrinsic parietal connections suggest some principles of organization, which 
may differ between animal and human brains. This divergence is particularly important in view of the simian-human similarities of the fronto-parietal connections, which are thought to have equivalent functions across the two species (Makris et al., 2005; Schmahmann \& Pandya, 2006). The difference in the intraparietal connectivity may explain interspecies divergence in functional activation studies of the parietal lobe and clinical manifestations unique to the human brain (Castiello, 2005; Caminiti et al., 2010).

Early animal model data identified two rostro-caudal streams of short connections within the parietal lobe (Pandya \& Seltzer, 1982). The dorso-medial stream connects superior areas of the postcentral gyrus to the superior parietal lobule (PE, $\mathrm{PEC}$ ) and precuneus (PGm), whereas the ventrolateral stream connects inferior areas of the postcentral gyrus to areas of the inferior parietal lobule (PG, PFG, PF, Opt). In our study, we identified, both in monkey and human brains, short bundles that may correspond to the connections forming the dorsal and ventral stream. The PSP, the PIG-SPL and PIG-PrCu mediate the dorsal connectivity, whereas the PIP-SMG, PIG-SMG and PAS mediate the connectivity of the ventral stream. The arrangement of these short connections has been interpreted as indicative of a serial organization of the two streams and a somatotopic organization extending beyond the postcentral gyrus into the posterior parietal cortex (Seltzer \& Pandya, 1978). While this may be valid for the segregation between a ventral stream for the mouth/tongue and an upper stream for the lower limbs, somatosensory information from the arm/hand seems to be processed along both streams as suggested by the common origin in the arm/ hand area of both PIP and PSP. Furthermore, the presence of large PIST pathways connecting the superior and inferior parietal lobules of both species is against the somatotopic segregation hypothesis. Other principles than somatotopy must therefore guide the arrangement of the intralobar parietal networks. We suggest that, similar to other brain regions (e.g., visual or motor), an integrative hypothesis may be at play in the parietal lobe. This hypothesis proposes that information, or processes that are correlated, tend to be mapped near each other in the cortex or overlap with each other (Meier, Aflalo, Kastner, \& Graziano, 2008). In the parietal lobe, the organization of the short connections could be shaped by the probability of different body parts working together. In general, high frequency tasks involving both arm/hand and mouth/tongue require converging processing in the ventral stream, whereas tasks with both arm/hand and leg/foot converge in the dorsal stream. This hypothesis is supported by fMRI activation studies that show functional clusters within the superior parietal (hand/wrist/foot) and inferior (hand/ wrist/arm/tongue) posterior parietal cortex (Meier et al., 2008).

Furthermore, in the human brain the connections between the dorsal postcentral gyrus and posterior regions of the inferior parietal lobule (posterior supramarginal gyrus and angular gyrus) represent a significant exception to the serial organization of the dorsal and ventral parietal streams proposed for the monkey brain. This evolutionary divergence may explain, for example, why the separation between a dorsal reach-to-grasp network and a ventral network for grasping proposed on the basis of animal studies may not directly apply to the human brain (Castiello, 2005; Davare et al., 2011). The direct communication through the PIP-AG highlights the importance of proprioceptive and tactile inputs in the acquisition of higher cognitive functions that are unique to our species and supported by the posterior regions of the inferior parietal cortex.

3.8.6. The special role of the inferior parietal lobule in humans Comparative cytoarchitectonic studies have shown a similar organization for the postcentral cortical areas (area 3, 1, 2) and anterior associative areas of the superior and inferior parietal lobule of the human and monkey brain. However, in more posterior parietal regions a divergence has been described with the emergence of cortical areas in humans that may not have an equivalent in the monkey brain (e.g., area 39 in the angular gyrus and other lateral areas of the superior parietal lobule) (Scheperjans et al., 2008).

Similarly, our tractography findings indicate a divergence in the connectional anatomy of the posterior regions of the inferior parietal lobule between monkey and human brains. The main difference consists in the direct access of these regions to somatosensory and proprioceptive information of the hand and arm (through the PIP-SMG and PIP-AG). The angular gyrus also receives high order auditory and visual inputs from the temporal and occipital cortex (Borra \& Luppino, 2017). The direct convergence of multimodal sensory information to an area that participates in episodic memory retrieval and language creates the ideal anatomical substrate for functions requiring the initiation of appropriate commands of motor sequences in tasks that involve knowledge of object use in time and space. This complexity of incoming information to the angular gyrus is matched with a reach output system consisting of short and long association tracts to ipsilateral parietal and frontal regions. The intraparietal connections to the superior parietal lobule are particular important to allocate attentional resources to complex functions of the angular gyrus, such as tool use, mathematical tasks (Dehaene et al., 2003), reading (Lobier, Peyrin, Pichat, Le Bas, \& Valdois, 2014), episodic memory retrieval (Cabeza et al., 2008; Sestieri et al., 2017), language and social communication (Catani \& Bambini, 2014). The role of the angular gyrus in many higher cognitive functions is certainly supramodal and could be related to its ability to apply internalised rules to hierarchical recursive stimuli to efficiently compress external information (Fischmeister, Martins, Beinssteiner, \& Fitch, 2017). This role may well apply to tool use, language, music, and social cognition.

In these terms, the angular gyrus represents one of the most complex hubs of the human brain and data on its connectivity allow to formulate some hypotheses that may explain functional evolutionary divergence and uniquely human parietal syndromes. Many disorders of the inferior parietal lobule can thus be reinterpreted as disconnection syndromes in which one or more of the several tracts projecting to the inferior parietal lobule are affected. The disconnection mechanism has been recently applied to the Gerstmann syndrome in which the symptoms of finger agnosia, left-right disorientation, acalculia and agraphia can present together or in partial combinations (e.g., finger agnosia and acalculia only) depending on the tracts affected by the lesion (Rusconi, Pinel, Dehaene, \& Kleinschmidt, 2010). The same interpretation can be applied to disorders of 
reading, writing, and social communication that have been associated with posterior parietal lesions (Catani \& Bambini, 2014; Catani, Dell'Acqua, Bizzi et al., 2012; Dragoy, Akinina, \& Dronkers, 2017).

The advantage of interpreting disorders of the parietal lobe as disconnection syndromes lies in the possibility of explaining those cases that present with either partial symptoms or atypical lesion location (Catani \& ffytche, 2005; Catani, Dell'Acqua, Bizzi et al., 2012). In the case of Gerstmann syndrome, for example, a complete disconnection of the tracts between angular and postcentral gyrus could manifest with prominent finger agnosia without significant deficits in leftright orientation, writing and calculation (Rusconi et al., 2009). In this cases the lesion may not involve directly the inferior parietal lobe and be located more in the deep white matter of the intraparietal sulcus.

\subsubsection{Limitations and future directions}

Two methodological aspects of our study deserve special attention as possible sources of errors. First, tractography reconstructions of the white matter pathways can generate artefacts, especially in those regions with complex fibre organization (Catani, Bodi, \& Dell'Acqua, 2012; Dell'Acqua \& Catani, 2012; Maier-Hein et al., 2017). In our study, we tried to partially overcome this problem by displaying only those connections that were consistently reproduced with both diffusion tensor and spherical deconvolution tractography methods and identified in our post-mortem dissections. Furthermore, U-shaped fibres have been described in the monkey brain using axonal tracing methods (Schmahmann \& Pandya, 2006) and we found a close correspondence to these studies for many of the virtually dissected tracts. Nevertheless, false positive and false negative reconstructions of $U$ shaped tracts are likely to occur with both diffusion tensor and spherical deconvolution approaches and validation of the proposed human pathways necessarily relies on future high resolution anatomical and electrophysiological studies.

Second, tractography provides only an approximate indication of the cortical projections. Many streamlines terminate in the gyral white matter and, therefore, it is difficult to infer an exact pattern of connectivity between cytoarchitectonically defined areas. We acknowledge that our approach for displaying cortical terminations of streamlines is tentative at this stage and for this reason we have avoided using more detailed cortical maps available for the human brain (Scheperjans et al., 2008). Future studies using high resolution diffusion data (Dell'Acqua, Bodi, Slater, Catani, \& Modo, 2013) combined with probabilistic cytoarchitectonic maps (Caspers et al., 2011) may reveal important details in this regard.

Finally, in this study we have not performed quantitative measures of the tracts that may reveal important information on possible differences between and within species. For example, previous studies in right handed healthy subjects have shown a variability in the leftward asymmetry of the Ushaped fibres connecting precentral with postcentral gyrus of the hand region (Catani, Bodi et al., 2012; Catani, Dell'Acqua, Bizzi et al., 2012; Catani, Dell'Acqua, Vergani et al., 2012) and a direct correlation between diffusion properties of these tracts and motor skill performance (Thompson et al., 2017). Our figures are from individual brains and therefore provide only an approximate indication of the anatomical location of the intraparietal fibres, which may not necessarily apply to the general population. Future quantitative studies of the inter-individual variability in tract volume, location and asymmetry are therefore necessary to give a comprehensive mapping of these fibres. Future correlative studies between tract anatomy and neuropsychological performance may also give important indications on the specific roles of each connection which at the moment remain highly speculative.

In conclusion, our study has described intralobar parietal connections in both monkey (macaque and vervet) and human brains. The results are indicative of the presence of both serial and parallel parietal networks, especially in the human brain, where other principles than somatotopy may have guided the development of cortico-cortical parietal connections. The unique arrangement of the connectivity of the angular gyrus may have contributed to the emergence of complex human functions. The proposed framework may help to interpret the results of functional imaging and clinicoanatomical correlation studies in humans and advance current knowledge of the distinctive functional role of the parietal lobe in our evolution.

\section{Acknowledgments}

Marco Catani is the recipient of the Wellcome Trust Investigator Award No. 103759/Z/14/Z. This work was also supported by a BBSRC grant to Kristine Krug (BB/H016902/1). The authors are grateful to Michel Thiebaut de Schotten and members of the NatBrainLab (www.natbrainlab.com) for their feedback. We would also like to thank the donor and her family, Kirsty Massetti and staff from the Dissecting Room, and Richard Wingate, Head of the Anatomy Department, King's College London for the post-mortem brain dissection.

\section{R E F E R E N C E S}

Andersson, J. L. R., \& Sotiropoulos, S. N. (2015). Non-parametric representation and prediction of single- and multi-shell diffusion-weighted MRI data using Gaussian processes. NeuroImage, 122, 166-176. https://doi.org/10.1016/ j.neuroimage.2015.07.067.

Bailey, P., \& Bonin, G. (1951). The isocortex of man. Illinois: Urbana: University of Illinois Press.

Besharati, S., Forkel, S. J., Kopelman, M., Solms, M., Jenkinson, P. M., \& Fotopoulou, A. (2016). Mentalizing the body: Spatial and social cognition in anosognosia for hemiplegia. Brain, 139(3), 971-985. https://doi.org/10.1093/ brain/awv390. Epub 2016 Jan 24.

Bonin, G., \& Bailey, P. (1947). The neocortex of Macaca mulatta. Urbana, IL: University of Illinois Press.

Borra, E., \& Luppino, G. (2017). Functional anatomy of the macaque temporo-parieto-frontal connectivity. Cortex (this issue).

Bruner, E., Lozano, M., Malafouris, L., Langbroek, M., Wynn, T., Coolidge, F. L., et al. (2014). Extended mind and visuo-spatial integration: Three hands for the Neandertal lineage. Journal of Anthropological Sciences = Rivista Di Antropologia: JASS, 92, 273-280. https://doi.org/10.4436/JASS.92009. 
Burton, H., Snyder, A. Z., Conturo, T. E., Akbudak, E., Ollinger, J. M., \& Raichle, M. E. (2002). Adaptive changes in early and late blind: A fMRI study of Braille reading. Journal of Neurophysiology, 87(1), 589-607.

Butterworth, B. (1999). The mathematical brain. London: Macmillan.

Cabeza, R., Ciaramelli, E., Olson, I. R., \& Moscovitch, M. (2008). The parietal cortex and episodic memory: An attentional account. Nature Reviews. Neuroscience, 9(8), 613-625. https://doi.org/ 10.1038/nrn2459.

Caminiti, R., Chafee, M. V., Battaglia-Mayer, A., Averbeck, B. B., Crowe, D. A., \& Georgopoulos, A. P. (2010). Understanding the parietal lobe syndrome from a neurophysiological and evolutionary perspective. The European Journal of Neuroscience, 31(12), 2320-2340. https://doi.org/10.1111/j.1460-9568.2010. 07291.x.

Campbell, A. W. (1905). Histological studies on the localisation of cerebral function. Cambridge: Cambridge University Press.

Caspers, S., Eickhoff, S. B., Rick, T., von Kapri, A., Kuhlen, T., Huang, R., et al. (2011). Probabilistic fibre tract analysis of cytoarchitectonically defined human inferior parietal lobule areas reveals similarities to macaques. NeuroImage, 58(2), 362-380. https://doi.org/10.1016/j.neuroimage.2011.06.027.

Caspers, S., Geyer, S., Schleicher, A., Mohlberg, H., Amunts, K., \& Zilles, K. (2006). The human inferior parietal cortex: Cytoarchitectonic parcellation and interindividual variability. NeuroImage, 33(2), 430-448. https://doi.org/10.1016/ j.neuroimage.2006.06.054.

Castiello, U. (2005). The neuroscience of grasping. Nature Reviews Neuroscience, 6(9), 726-736. https://doi.org/10.1038/nrn1744.

Catani, M., \& Bambini, V. (2014). A model for social communication and language evolution and development (SCALED). Current Opinion in Neurobiology, 28, 165-171. https:// doi.org/10.1016/j.conb.2014.07.018.

Catani, M., Bodi, I., \& Dell'Acqua, F. (2012). Comment on "The geometric structure of the brain fiber pathways." Science (New York, N.Y.), 337(6102), 1605. https://doi.org/10.1126/science. 1223425.

Catani, M., \& de Schotten, M. T. (2012). Atlas of human brain connections. Oxford University Press.

Catani, M., Dell'Acqua, F., Bizzi, A., Forkel, S. J., Williams, S. C., Simmons, A., et al. (2012). Beyond cortical localization in clinico-anatomical correlation. Cortex, 48(10), 1262-1287. https://doi.org/10.1016/j.cortex.2012.07.001.

Catani, M., Dell'Acqua, F., Vergani, F., Malik, F., Hodge, H., Roy, P., et al. (2012). Short frontal lobe connections of the human brain. Cortex, 48(2), 273-291. https://doi.org/10.1016/ j.cortex.2011.12.001.

Catani, M., \& ffytche, D. H. (2005). The rises and falls of disconnection syndromes. Brain, 128(10), 2224-2239. https:// doi.org/10.1093/brain/awh622.

Cavada, C., \& Goldman-Rakic, P. S. (1989). Posterior parietal cortex in rhesus monkey: I. Parcellation of areas based on distinctive limbic and sensory corticocortical connections. The Journal of Comparative Neurology, 287(4), 393-421. https://doi.org/10.1002/ cne.902870402.

Corbetta, M., Kincade, M. J., Lewis, C., Snyder, A. Z., \& Sapir, A. (2005). Neural basis and recovery of spatial attention deficits in spatial neglect. Nature Neuroscience, 8(11), 1603-1610. https:// doi.org/10.1038/nn1574.

Corbetta, M., \& Shulman, G. L. (2002). Control of goal-directed and stimulus-driven attention in the brain. Nature Reviews Neuroscience, 3(3), 201-215. https://doi.org/10.1038/nrn755.

Corbetta, M., \& Shulman, G. L. (2011). Spatial neglect and attention networks. Annual Review of Neuroscience, 34(1), 569-599. https://doi.org/10.1146/annurev-neuro-061010113731.

de Crespigny, A. J., D'Arceuil, H. E., Maynard, K. I., He, J., McAuliffe, D., Norbash, A., et al. (2005). Acute studies of a new primate model of reversible middle cerebral artery occlusion. Journal of Stroke and Cerebrovascular Diseases, 14(2), 80-87. https://doi.org/10.1016/j.jstrokecerebrovasdis.2004.12.005.

Critchley, M. (1953). The parietal lobes. London: Edward Arnold.

Culham, J. C., \& Kanwisher, N. G. (2001). Neuroimaging of cognitive functions in human parietal cortex. Current Opinion in Neurobiology, 11(2), 157-163.

Davare, M., Kraskov, A., Rothwell, J. C., \& Lemon, R. N. (2011). Interactions between areas of the cortical grasping network. Current Opinion in Neurobiology, 21(4), 565-570. https://doi.org/ 10.1016/j.conb.2011.05.021.

Dehaene, S., Piazza, M., Pinel, P., \& Cohen, L. (2003). Three parietal circuits for number processing. Cognitive Neuropsychology, 20(3), 487-506. https://doi.org/10.1080/02643290244000239.

Dell'Acqua, F., Bodi, I., Slater, D., Catani, M., \& Modo, M. (2013). MR diffusion histology and micro-tractography reveal mesoscale features of the human cerebellum. Cerebellum (London, England), 12(6), 923-931. https://doi.org/10.1007/s12311-013-0503-x.

Dell'Acqua, F., \& Catani, M. (2012). Structural human brain networks: Hot topics in diffusion tractography. Current Opinion in Neurology, 25(4), 375-383. https://doi.org/10.1097/ WCO.0b013e328355d544.

Dell'acqua, F., Scifo, P., Rizzo, G., Catani, M., Simmons, A., Scotti, G., et al. (2010). A modified damped Richardson-Lucy algorithm to reduce isotropic background effects in spherical deconvolution. NeuroImage, 49(2), 1446-1458. https://doi.org/ 10.1016/j.neuroimage.2009.09.033.

Dell'Acqua, F., Simmons, A., Williams, S. C. R., \& Catani, M. (2013b). Can spherical deconvolution provide more information than fiber orientations? Hindrance modulated orientational anisotropy, a true-tract specific index to characterize white matter diffusion. Human Brain Mapping, 34(10), 2464-2483. https://doi.org/10.1002/hbm. 22080.

Dominici, F., Popa, T., Ginanneschi, F., Mazzocchio, R., \& Rossi, A. (2005). Cortico-motoneuronal output to intrinsic hand muscles is differentially influenced by static changes in shoulder positions. Experimental Brain Research Experimentelle Hirnforschung Experimentation Cerebrale, 164(4), 500-504. https://doi.org/10.1007/s00221-005-2270-5.

Donkelaar, H. J., Broman, J., Neumann, P. E., Puelles, L., Riva, A., Tubbs, R. S., et al. (2017). Towards a Terminologia Neuroanatomica. Clinical Anatomy (New York, N.Y.), 30(2), 145-155. https://doi.org/10.1002/ca.22809.

Dragoy, O., Akinina, Y., \& Dronkers, N. F. (2017). Toward a functional neuroanatomy of semantic aphasia: A history and ten new cases. Cortex, (this issue).

Dyrby, T. B., Baaré, W. F. C., Alexander, D. C., Jelsing, J., Garde, E., \& Søgaard, L. V. (2011). An ex vivo imaging pipeline for producing high-quality and high-resolution diffusionweighted imaging datasets. Human Brain Mapping, 32(4), 544-563. https://doi.org/10.1002/hbm.21043.

D'Arceuil, H. E., Westmoreland, S., \& de Crespigny, A. J. (2007). An approach to high resolution diffusion tensor imaging in fixed primate brain. NeuroImage, 35(2), 553-565. https://doi.org/ 10.1016/j.neuroimage.2006.12.028.

Engelen, T., de Graaf, T. A., Sack, A. T., \& de Gelder, B. (2015). A causal role for inferior parietal lobule in emotion body perception. Cortex, 73, 195-202. https://doi.org/10.1016/ j.cortex.2015.08.013.

Fattori, P., Breveglieri, R., Marzocchi, N., Filippini, D., Bosco, A., \& Galletti, C. (2009). Hand orientation during reach-to-grasp movements modulates neuronal activity in the medial posterior parietal area V6A. The Journal of Neuroscience, 29(6), 1928-1936. https://doi.org/10.1523/ JNEUROSCI.4998-08.2009.

Fischl, B., Salat, D. H., van der Kouwe, A. J. W., Makris, N., Ségonne, F., Quinn, B. T., et al. (2004). Sequence-independent segmentation of magnetic resonance images. NeuroImage, 
23(Suppl. 1), S69-S84. https://doi.org/10.1016/ j.neuroimage.2004.07.016.

Fischmeister, F. P., Martins, M. J., Beinssteiner, R., \& Fitch, T. W. (2017). Self-similarity and recursion as default modes in human cognition. Cortex, (this issue).

de Gelder, B., Tamietto, M., Pegna, A. J., \& Van den Stock, J. (2015). Visual imagery influences brain responses to visual stimulation in bilateral cortical blindness. Cortex, 72, 15-26. https://doi.org/10.1016/j.cortex.2014.11.009.

Geng, J. J., \& Mangun, G. R. (2011). Right temporoparietal junction activation by a salient contextual cue facilitates target discrimination. NeuroImage, 54(1), 594-601. https://doi.org/ 10.1016/j.neuroimage.2010.08.025.

Geschwind, N. (1965). Disconnexion syndromes in animals and man. I. Brain, 88(2), 237-294.

Gillebert, C. R., Mantini, D., Thijs, V., Sunaert, S., Dupont, P., \& Vandenberghe, R. (2011). Lesion evidence for the critical role of the intraparietal sulcus in spatial attention. Brain, 134(Pt 6), 1694-1709. https://doi.org/10.1093/brain/awr085.

Glass, L., Krueger, F., Solomon, J., Raymont, V., \& Grafman, J. (2013). Mental paper folding performance following penetrating traumatic brain injury in combat veterans: A lesion mapping study. Cerebral Cortex (New York, N.Y.: 1991), 23(7), 1663-1672. https://doi.org/10.1093/cercor/bhs153.

Goldenberg, G. (2013). Apraxia. Oxford University Press.

Grafton, S. T. (2010). The cognitive neuroscience of prehension: Recent developments. Experimental Brain Research, 204(4), 475-491. https://doi.org/10.1007/s00221-010-2315-2.

Grefkes, C., \& Fink, G. R. (2005). The functional organization of the intraparietal sulcus in humans and monkeys. Journal of Anatomy, 207(1), 3-17. https://doi.org/10.1111/j.14697580.2005.00426.x.

Guevara, P., Poupon, C., Rivière, D., Cointepas, Y., Descoteaux, M., Thirion, B., et al. (2011). Robust clustering of massive tractography datasets. NeuroImage, 54(3), 1975-1993. https:// doi.org/10.1016/j.neuroimage.2010.10.028.

Guevara, M., Román, C., Houenou, J., Duclap, D., Poupon, C., Mangin, J. F., et al. (2017). Reproducibility of superficial white matter tracts using diffusion-weighted imaging tractography. NeuroImage, 147, 703-725. https://doi.org/10.1016/j.neuroimage. 2016.11.066.

Hänggi, J., Vitacco, D. A., Hilti, L. M., Luechinger, R., Kraemer, B., \& Brugger, P. (2017). Structural and functional hyperconnectivity within the sensorimotor system in xenomelia. Brain and Behavior, 7(3), e00657. https://doi.org/10.1002/brb3.657.

Hikosaka, O., Tanaka, M., Sakamoto, M., \& Iwamura, Y. (1985). Deficits in manipulative behaviors induced by local injections of muscimol in the first somatosensory cortex of the conscious monkey. Brain Research, 325(1-2), 375-380.

Hilti, L. M., Hänggi, J., Vitacco, D. A., Kraemer, B., Palla, A., Luechinger, R., et al. (2012). The desire for healthy limb amputation: Structural brain correlates and clinical features of xenomelia. Brain, 136(1), 318-329. https://doi.org/10.1093/ brain/aws316.

Husain, M., Mannan, S. K., Hodgson, T. L., Wojciulik, E., Driver, J., \& Kennard, C. (2001). Impaired spatial working memory across saccades contributes to abnormal search in parietal neglect. Brain, 124(Pt 5), 941-952.

Jeannerod, M. (2006). Motor cognition. Oxford University Press.

Jenkinson, M., Bannister, P., Brady, M., \& Smith, S. (2002). Improved optimization for the robust and accurate linear registration and motion correction of brain images. NeuroImage, 17(2), 825-841.

Jones, E. G., \& Powell, T. P. (1969). Connexions of the somatic sensory cortex of the rhesus monkey. I. Ipsilateral cortical connexions. Brain, 92(3), 477-502.
Jones, E. G., \& Powell, T. P. (1970). An anatomical study of converging sensory pathways within the cerebral cortex of the monkey. Brain, 93(4), 793-820.

Karnath, H. O., \& Perenin, M. T. (2005). Cortical control of visually guided reaching: Evidence from patients with optic ataxia. Cerebral Cortex, 15(10), 1561-1569. https://doi.org/10.1093/ cercor/bhi034.

Large, I., Bridge, H., Ahmed, B., Clare, S., Kolasinski, J., Lam, W. W., et al. (2016). Individual differences in the alignment of structural and functional markers of the V5/MT complex in primates. Cerebral Cortex, 26(10), 3928-3944. https://doi.org/10.1093/cercor/bhw180.

Lecce, F., Walsh, V., Didino, D., \& Cappelletti, M. (2015). 'How many' and 'how much' dissociate in the parietal lobe. Cortex, 73, 73-79. https://doi.org/10.1016/j.cortex.2015.08.007.

Lewis, J. W. (2006). Cortical networks related to human use of tools. The Neuroscientist, 12(3), 211-231. https://doi.org/10.1177/ 1073858406288327.

Lobier, M. A., Peyrin, C., Pichat, C., Le Bas, J.-F., \& Valdois, S. (2014) Visual processing of multiple elements in the dyslexic brain: Evidence for a superior parietal dysfunction. Frontiers in Human Neuroscience, 8(476), 479. https://doi.org/10.3389/fnhum.2014. 00479.

Ludwig, E., \& Klingler, J. (1956). Atlas cerebri humani. The inner structure of the brain demonstrated on the basis of macroscopical preparations. Boston: Little Brown.

Magrassi, L., Bongetta, D., Bianchini, S., Berardesca, M., \& Arienta, C. (2010). Central and peripheral components of writing critically depend on a defined area of the dominant superior parietal gyrus. Brain Research, 1346, 145-154. https:// doi.org/10.1016/j.brainres.2010.05.046.

Maier-Hein, K. H., Neher, P. F., Houde, J.-C., Côté, M.-A., Garyfallidis, E., Zhong, J., et al. (2017). The challenge of mapping the human connectome based on diffusion tractography. Nature Communications, 8(1), 1349. https://doi. org/10.1038/s41467-017-01285-x.

Makris, N., Kennedy, D. N., McInerney, S., Sorensen, A. G., Wang, R. P., Caviness, V. S., et al. (2005). Segmentation of subcomponents within the superior longitudinal fascicle in humans: A quantitative, in vivo, DT-MRI study. Cerebral Cortex (New York, N.Y.: 1991), 15(6), 854-869. https://doi.org/10.1093/ cercor/bhh186.

Martin, J. A., Karnath, H. O., \& Himmelbach, M. (2015). Revisiting the cortical system for peripheral reaching at the parietooccipital junction. Cortex, 64, 363-379. https://doi.org/10.1016/ j.cortex.2014.11.012.

Martin, M., Nitschke, K., Beume, L., Dressing, A., Bühler, L. E., Ludwig, V. M., et al. (2016). Brain activity underlying tool-related and imitative skills after major left hemisphere stroke. Brain, 139(Pt 5), 1497-1516. https://doi.org/10.1093/brain/aww035.

Meier, J. D., Aflalo, T. N., Kastner, S., \& Graziano, M. S. A. (2008). Complex organization of human primary motor cortex: A high-resolution fMRI study. Journal of Neurophysiology, 100(4), 1800-1812. https://doi.org/10.1152/jn.90531.2008.

Moffett, A., Ettlinger, G., Morton, H. B., \& Piercy, M. F. (1967). Tactile discrimination performance in the Monkey: The effect of ablation of various subdivisions of posterior parietal cortex. Cortex, 3(1), 59-96. https://doi.org/10.1016/S0010-9452(67) 80006-6.

Mountcastle, V. B. (1995). The parietal system and some higher brain functions. Cerebral Cortex (New York, N.Y.: 1991), 5(5), 377-390.

Pandya, D. N., \& Seltzer, B. (1982). Intrinsic connections and architectonics of posterior parietal cortex in the rhesus monkey. The Journal of Comparative Neurology, 204(2), 196-210. https://doi.org/10.1002/cne.902040208. 
Papez, J. W. (1929). Comparative neurology; a manual and text for the study of the nervous system of vertebrates. NY: Thomas Y. Crowell Publishers.

Parlatini, V., Radua, J., Dell'Acqua, F., Leslie, A., Simmons, A., Murphy, D. G., et al. (2017). Functional segregation and integration within fronto-parietal networks. NeuroImage, 146, 367-375. https://doi.org/10.1016/j.neuroimage.2016.08.031.

Patel, G. H., Yang, D., Jamerson, E. C., Snyder, L. H., Corbetta, M., \& Ferrera, V. P. (2015). Functional evolution of new and expanded attention networks in humans. Proceedings of the National Academy of Sciences of the United States of America, 112(30), 9454-9459. https://doi.org/10.1073/pnas.1420395112.

Paxinos, G., Huang, X. F., \& Toga, A. W. (2000). The rhesus monkey brain in stereotaxic coordinates.

Pisella, L., Binkofski, F., Lasek, K., Toni, I., \& Rossetti, Y. (2006). No double-dissociation between optic ataxia and visual agnosia: Multiple sub-streams for multiple visuo-manual integrations. Neuropsychologia, 44(13), 2734-2748. https://doi.org/10.1016/ j.neuropsychologia.2006.03.027.

Planton, S., Jucla, M., Roux, F.-E., \& Démonet, J.-F. (2013). The "handwriting brain": A meta-analysis of neuroimaging studies of motor versus orthographic processes. Cortex, 49(10), 2772-2787. https://doi.org/10.1016/j.cortex.2013.05.011.

Purcell, J. J., Turkeltaub, P. E., Eden, G. F., \& Rapp, B. (2011). Examining the central and peripheral processes of written word production through meta-analysis. Frontiers in Psychology, 2, 239. https://doi.org/10.3389/fpsyg.2011.00239.

Rapp, B., Purcell, J., Hillis, A. E., Capasso, R., \& Miceli, G. (2016). Neural bases of orthographic long-term memory and working memory in dysgraphia. Brain, 139(Pt 2), 588-604. https:// doi.org/10.1093/brain/awv348.

Rizzolatti, G., \& Matelli, M. (2003). Two different streams form the dorsal visual system: Anatomy and functions. Experimental Brain Research Experimentelle Hirnforschung Experimentation Cerebrale, 153(2), 146-157. https://doi.org/10.1007/s00221-003-1588-0.

Rozzi, S., Calzavara, R., Belmalih, A., Borra, E., Gregoriou, G. G., Matelli, M., et al. (2006). Cortical connections of the inferior parietal cortical convexity of the macaque monkey. Cerebral Cortex, 16(10), 1389-1417. https://doi.org/10.1093/cercor/bhj076.

Rozzi, S., Ferrari, P. F., Bonini, L., Rizzolatti, G., \& Fogassi, L. (2008). Functional organization of inferior parietal lobule convexity in the macaque monkey: Electrophysiological characterization of motor, sensory and mirror responses and their correlation with cytoarchitectonic areas. The European Journal of Neuroscience, 28(8), 1569-1588. https://doi.org/10.1111/j.14609568.2008.06395.x.

Rusconi, E., Pinel, P., Dehaene, S., \& Kleinschmidt, A. (2010). The enigma of Gerstmann's syndrome revisited: a telling tale of the vicissitudes of neuropsychology. Brain: a Journal of Neurology, 133(2), 320-332. https://doi.org/10.1093/brain/awp281.

Rusconi, E., Pinel, P., Eger, E., LeBihan, D., Thirion, B., Dehaene, S., et al. (2009). A disconnection account of Gerstmann syndrome: Functional neuroanatomy evidence. Annals of Neurology, 66(5), 654-662. https://doi.org/10.1002/ana.21776.

Rushworth, M. F. S., Behrens, T. E. J., \& Johansen-Berg, H. (2006). Connection patterns distinguish 3 regions of human parietal cortex. Cerebral Cortex (New York, N.Y.: 1991), 16(10), 1418-1430.

Sadato, N., Pascual-Leone, A., Grafman, J., Deiber, M. P., Ibañez, V., \& Hallett, M. (1998). Neural networks for Braille reading by the blind. Brain, 121(Pt 7), 1213-1229.

Sakata, H., Takaoka, Y., Kawarasaki, A., \& Shibutani, H. (1973). Somatosensory properties of neurons in the superior parietal cortex (area 5) of the rhesus monkey. Brain Research, 64, 85-102.

Scheperjans, F., Hermann, K., Eickhoff, S. B., Amunts, K., Schleicher, A., \& Zilles, K. (2008). Observer-independent cytoarchitectonic mapping of the human superior parietal cortex. Cerebral Cortex (New York, N.Y.: 1991), 18(4), 846-867. https://doi.org/10.1093/cercor/bhm116.
Schmahmann, J. D., \& Pandya, D. N. (2006). Fiber pathways of the brain. Oxford: Oxford University Press.

Seelke, A. M., Padberg, J. J., Disbrow, E., Purnell, S. M., Recanzone, G., \& Krubitzer, L. (2012). Topographic Maps within Brodmann's area 5 of macaque monkeys. Cerebral Cortex, 22(8), 1834-1850. https://doi.org/10.1093/cercor/bhr257.

Seltzer, B., \& Pandya, D. N. (1978). Afferent cortical connections and architectonics of the superior temporal sulcus and surrounding cortex in the rhesus monkey. Brain Research, 149(1), 1-24.

Sestieri, C., Shulman, G. L., \& Corbetta, M. (2017). The contribution of the human posterior parietal cortex to episodic memory. Nature Reviews. Neuroscience, 18(3), 183-192. https://doi.org/ 10.1038/nrn.2017.6.

Shattuck, D. W., \& Leahy, R. M. (2002). BrainSuite: An automated cortical surface identification tool. Medical Image Analysis, 6(2), 129-142.

Shulman, G. L., Astafiev, S. V., McAvoy, M. P., d'Avossa, G., \& Corbetta, M. (2007). Right TPJ deactivation during visual search: Functional significance and support for a filter hypothesis. Cerebral Cortex, 17(11), 2625-2633. https://doi.org/ 10.1093/cercor/bhl170.

Tamietto, M., Cauda, F., Celeghin, A., Diano, M., Costa, T., Cossa, F. M., et al. (2015). Once you feel it, you see it: Insula and sensory-motor contribution to visual awareness for fearful bodies in parietal neglect. Cortex, 62, 56-72. https://doi.org/ 10.1016/j.cortex.2014.10.009.

Thiebaut de Schotten, M., Dell'Acqua, F., Forkel, S. J., Simmons, A., Vergani, F., Murphy, D. G., et al. (2011). A lateralized brain network for visuospatial attention. Nature Neuroscience, 14(10), 1245-1246. https://doi.org/10.1038/nn.2905.

Thiebaut de Schotten, M., Dell'Acqua, F., Valabregue, R., \& Catani, M. (2012). Monkey to human comparative anatomy of the frontal lobe association tracts. Cortex, 48(1), 82-96. https:// doi.org/10.1016/j.cortex.2011.10.001.

Thompson, A., Murphy, D., Dell'Acqua, F., Ecker, C., McAlonan, G., Howells, H., et al. (2017). Impaired communication between the motor and somatosensory homunculus is associated with poor manual dexterity in autism spectrum disorder. Biological Psychiatry, 81(3), 211-219. https://doi.org/10.1016/j.biopsych. 2016.06.020. Epub 2016 Jul 1.

Tian, X., Zarate, J. M., \& Poeppel, D. (2016). Mental imagery of speech implicates two mechanisms of perceptual reactivation. Cortex, 77, 1-12. https://doi.org/10.1016/j.cortex.2016.01.002.

Tsutsui, K., Jiang, M., Sakata, H., \& Taira, M. (2003). Short-term memory and perceptual decision for three-dimensional visual features in the caudal intraparietal sulcus (Area CIP). Journal of Neuroscience, 23(13), 5486-5495.

Tyler, S. C., Dasgupta, S., Agosta, S., Battelli, L., \& Grossman, E. D. (2015). Functional connectivity of parietal cortex during temporal selective attention. Cortex, 65, 195-207. https:// doi.org/10.1016/j.cortex.2015.01.015.

Umarova, R. M. (2017). Adapting the concepts of brain and cognitive reserve to post-stroke cognitive deficits: Implications for understanding neglect. Cortex, (this issue).

Verhagen, L., Dijkerman, H. C., Grol, M. J., \& Toni, I. (2008). Perceptuo-motor interactions during prehension movements. The Journal of Neuroscience, 28(18), 4726-4735. https://doi.org/ 10.1523/JNEUROSCI.0057-08.2008.

Vossel, S., Geng, J. J., \& Fink, G. R. (2014). Dorsal and ventral attention systems: Distinct neural circuits but collaborative roles. The Neuroscientist, 20(2), 150-159. https://doi.org/10.1177/ 1073858413494269.

Warren, M. S. (1944). The functional organization of the cerebral cortex. Physiological Reviews, 24(3), 390-407.

Watson, C. E., \& Buxbaum, L. J. (2015). A distributed network critical for selecting among tool-directed actions. Cortex, 65, 65-82. https://doi.org/10.1016/j.cortex.2015.01.007. 
Wen, X., Yao, L., Liu, Y., \& Ding, M. (2012). Causal interactions in attention networks predict behavioral performance. The Journal of Neuroscience, 32(4), 1284-1292. https://doi.org/ 10.1523/JNEUROSCI.2817-11.2012.

Wolbers, T., Schoell, E. D., \& Büchel, C. (2006). The predictive value of white matter organization in posterior parietal cortex for spatial visualization ability. NeuroImage, 32(3), 1450-1455. https://doi.org/10.1016/j.neuroimage.2006.05.011.

Wood, D. K., Chouinard, P. A., Major, A. J., \& Goodale, M. A. (2017). Sensitivity to biomechanical limitations during postural decision-making depends on the integrity of posterior superior parietal cortex. Cortex, (this issue).

Zacks, J. M. (2008). Neuroimaging studies of mental rotation: A meta-analysis and review. Journal of Cognitive Neuroscience, 20(1), 1-19. https://doi.org/10.1162/jocn.2008.20.1.1.

Zhang, Y., Zhang, J., Oishi, K., Faria, A. V., Jiang, H., Li, X., et al. (2010). Atlas-guided tract reconstruction for automated and comprehensive examination of the white matter anatomy. NeuroImage, 52(4), 1289-1301. https://doi.org/10.1016/ j.neuroimage.2010.05.049. 